\title{
A multifunctional GH39 glycoside hydrolase from the anaerobic gut fungus Orpinomyces sp. strain C1A
}

\author{
Jessica M Morrison ${ }^{1}{ }^{,}$Mostafa S Elshahed ${ }^{1}$, Noha Youssef ${ }^{\text {Corresp. } 1}$ \\ ${ }^{1}$ Department of Microbiology and Molecular Genetics, Oklahoma State University, Stillwater, OK, USA \\ Corresponding Author: Noha Youssef \\ Email address: noha@okstate.edu
}

Background. The anaerobic gut fungi (phylum Neocallimastigomycota) represent a promising source of novel lignocellulolytic enzymes. Here, we report on the cloning, expression, and characterization of a glycoside hydrolase family 39 (GH39) enzyme (Bgxg1) that is highly transcribed by the anaerobic fungus Orpinomyces sp. strain C1A under different growth conditions. This represents the first study of a GH39family enzyme from the anaerobic fungi.

Methods. Using enzyme activity assays, we performed a biochemical characterization of Bgxg1 on a variety of substrates over a wide range of $\mathrm{pH}$ and temperature values to identify the optimal enzyme conditions and the specificity of the enzyme. In addition, substrate competition studies and comparative modeling efforts were completed.

Results. Contrary to the narrow range of activities ( $\beta$-xylosidase or $\alpha$-L-iduronidase) observed in previously characterized GH39 enzymes, Bgxg1 is unique in that it is multifunctional, exhibiting strong $\beta$ xylosidase, $\beta$-glucosidase, $\beta$-galactosidase activities $(11.5 \pm 1.2,73.4 \pm 7.15$, and $54.6 \pm 2.26 \mathrm{U} / \mathrm{mg}$, respectively) and a weak xylanase activity $(10.8 \pm 1.25 \mathrm{U} / \mathrm{mg})$, as compared to previously characterized enzymes. Further, Bgxg1 possesses extremely high affinity (as evident by the lowest $K_{m}$ values), compared to all previously characterized $\beta$-glucosidases, $\beta$-galactosidases, and xylanases. Physiological characterization revealed that $\mathrm{Bgxg} 1$ is active over a wide range of $\mathrm{pH}(3-8$, optimum 6$)$ and temperatures $\left(25-60^{\circ} \mathrm{C}\right.$, optimum $\left.39^{\circ} \mathrm{C}\right)$, and possesses excellent temperature and thermal stability. Substrate competition assays suggest that all observed activities occur at a single active site. Using comparative modeling and bioinformatics approaches, we putatively identified ten amino acid differences between Bgxg1 and previously biochemically characterized GH39 $\beta$-xylosidases that we speculate could impact active site architecture, size, charge, and/or polarity.

Discussion. Collectively, the unique capabilities and multi-functionality of Bgxg1 render it an excellent candidate for inclusion in enzyme cocktails mediating cellulose and hemicellulose saccharification from lignocellulosic biomass. 
A multifunctional GH39 glycoside hydrolase from the anaerobic gut fungus Orpinomyces sp. strain C1A

Keywords: Anaerobic gut fungi, GH39, $\beta$-glucosidase, $\beta$-xylosidase, $\beta$-galactosidase

Short Title: Triple oligosaccharide hydrolase activity from a rumen fungus GH39 enzyme

\section{*Corresponding author.}

Noha H. Youssef, Ph.D.

1110 S. Innovation way, Stillwater, Oklahoma 74074 USA.

Phone: 1-405-744-1192, Fax. 1-405-744-1112

Noha@Okstate.edu 


\section{Abstract}

41 Background. The anaerobic gut fungi (phylum Neocallimastigomycota) represent a promising

42 source of novel lignocellulolytic enzymes. Here, we report on the cloning, expression, and

43 characterization of a glycoside hydrolase family 39 (GH39) enzyme (Bgxg1) that is highly

44 transcribed by the anaerobic fungus Orpinomyces sp. strain C1A under different growth

45 conditions. This represents the first study of a GH39-family enzyme from the anaerobic fungi.

46 Methods. Using enzyme activity assays, we performed a biochemical characterization of Bgxg1

47 on a variety of substrates over a wide range of $\mathrm{pH}$ and temperature values to identify the optimal

48 enzyme conditions and the specificity of the enzyme. In addition, substrate competition studies

49 and comparative modeling efforts were completed.

50 Results. Contrary to the narrow range of activities ( $\beta$-xylosidase or $\alpha$-L-iduronidase) observed in

51 previously characterized GH39 enzymes, Bgxg1 is unique in that it is multifunctional, exhibiting

52 strong $\beta$-xylosidase, $\beta$-glucosidase, $\beta$-galactosidase activities $(11.5 \pm 1.2,73.4 \pm 7.15$, and $54.6 \pm$

$532.26 \mathrm{U} / \mathrm{mg}$, respectively) and a weak xylanase activity $(10.8 \pm 1.25 \mathrm{U} / \mathrm{mg})$, as compared to

54 previously characterized enzymes. Further, Bgxg1 possesses extremely high affinity (as evident

55 by the lowest $K_{m}$ values), compared to all previously characterized $\beta$-glucosidases, $\beta$ -

56 galactosidases, and xylanases. Physiological characterization revealed that Bgxg1 is active over a

57 wide range of $\mathrm{pH}\left(3-8\right.$, optimum 6) and temperatures $\left(25-60^{\circ} \mathrm{C}\right.$, optimum $\left.39^{\circ} \mathrm{C}\right)$, and possesses

58 excellent temperature and thermal stability. Substrate competition assays suggest that all

59 observed activities occur at a single active site. Using comparative modeling and bioinformatics

60 approaches, we putatively identified ten amino acid differences between Bgxg1 and previously

61 biochemically characterized GH39 $\beta$-xylosidases that we speculate could impact active site

62 architecture, size, charge, and/or polarity. 
63 Discussion. Collectively, the unique capabilities and multi-functionality of Bgxg1 render it an

64 excellent candidate for inclusion in enzyme cocktails mediating cellulose and hemicellulose 65 saccharification from lignocellulosic biomass. 


\section{Introduction}

67 The production of biofuels from lignocellulosic biomass is a global priority, necessitated by the

68 continuous depletion of recoverable fossil fuel reserves, the deleterious impact of fossil fuels on

69 air quality, as well as their contribution to global climate change (Hill et al. 2006; National

70 Research Council 2011; Ragauskas et al. 2006). Lignocellulosic biomass represents a vastly

71 underutilized and largely untapped source of energy, and its mass utilization for biofuel

72 production is one of the goals enacted by the U.S. Congress-implemented Renewable Fuel

73 Standard (RFS), aiming to generate 16 billion gallons of biofuel from lignocellulosic sources by

742022 (National Research Council 2011).

75 The most frequently used method of biofuel production from lignocellulosic biomass is

76 the enzymatic conversion of cellulose and hemicellulose polymers into sugar monomers/

77 oligomers that could subsequently be converted into biofuels using dedicated sugar metabolizers

78 (Elshahed 2010; Hill et al. 2006; Kumar et al. 2008). Historically, enzymatic cocktails designed

79 for the breakdown of lignocellulosic biomass focused primarily on cellulose degradation, due to

80 its relative structural simplicity and uniformity across all types of plant biomass. Nevertheless,

81 the hemicellulose components in lignocellulosic biomass should not be ignored, as hemicellulose

82 represents $20-35 \%$ of the composition of lignocellulosic biomass (Liu et al. 2008). Unlike

83 cellulose, plant hemicelluloses are structurally more complex, with multiple types of major

84 hemicelluloses (arabinoxylans/ glucuronoarabinoxylans, glucomannans/galactoglucomannans,

85 mixed glucans, and xyloglucans) present in various plants (Scheller \& Ulvskov 2010). The most

86 common type of hemicellulose are the arabinoxylans/ glucuronoarabinoxylans that possess a

87 structural backbone of $\beta$-1,4-linked xylose units (Scheller \& Ulvskov 2010). Xylan degradation

88 requires the consorted action of the endo-acting- $\beta-1,4-x y l a n a s e s$ and the oligosaccharide 
89 depolymerizing $\beta$-xylosidases, among other enzymes (Elshahed 2010; Scheller \& Ulvskov 90 2010).

The identification and characterization of novel enzymes and enzyme cocktails with

92 superior lignocellulosic biomass saccharification properties (e.g. high substrate affinity and 93 specific activity, activity retention at a wide range of $\mathrm{pH}$ and temperatures, and thermal and $\mathrm{pH}$

94 stability) signify essential thrusts in biofuel research. Members of the anaerobic gut fungi 95 (phylum Neocallimastigomycota) represent a promising, and largely untapped, source of 96 biomass-degrading enzymes (Ljungdahl 2008; Wang et al. 2013). Members of the

97 Neocallimastigomycota are found in the herbivorous gut, where they are responsible for the 98 initial colonization and degradation of plant materials ingested by their hosts (Ljungdahl 2008;

99 Wang et al. 2013). While anaerobic gut fungi were initially discovered in sheep, they have since 100 been found in the rumen and alimentary tracks of both ruminant and non-ruminant mammalian 101 and reptilian herbivores (Youssef et al. 2013). The anaerobic gut fungi are excellent biomass 102 degraders, capable of fast, efficient, and simultaneous degradation of the cellulolytic and 103 hemicellulolytic fraction of various plants, including most common lignocellulosic biomass 104 substrates (e.g. Corn Stover, Switchgrass, Sorghum, Energy Cane, and Alfalfa) (Borneman et al. 105 1989; Harhangi et al. 2003; Liggenstoffer et al. 2014; Youssef et al. 2013). Nevertheless, there 106 have been extensive efforts dedicated to bioprospecting novel cellulases and hemicellulases from 107 aerobic fungi (such as Aspergillus (Kumar \& Ramon 1996; vanPeij et al. 1997), Trichoderma 108 (Matsuo \& Yasui 1984)), anaerobic prokaryotes (such as Clostridium (Bronnenmeier \& 109 Staudenbauer 1988) and Thermoanaerobacterium (Shao et al. 2011)) and metagenomic sequence 110 data (Brennan et al. 2004; Hess et al. 2011); in comparison to these numerous efforts, the 
111 identification, expression, and characterization of such enzymes from anaerobic fungi has not

112 been as well represented in the literature (Borneman et al. 1989; Harhangi et al. 2003).

113 We aim to explore the utility of the anaerobic gut fungus Orpinomyces sp. strain C1A

114 (henceforth referred to as C1A) as a novel source of lignocellulolytic enzymes. C1A is an isolate

115 from the feces of an angus steer on cellobiose-switchgrass media (Youssef et al. 2013). Our

116 approach depends on implementing a transcriptomics-guided strategy to identify carbohydrate-

117 active enzymes (CAZyme) transcripts that are highly expressed by $\mathrm{C} 1 \mathrm{~A}$ when grown on

118 lignocellulosic biomass substrates as candidates for cloning, expression, and characterization.

119 Here, we describe our efforts in cloning, expression, and characterization of one such enzyme: a

120 GH39 transcript bioinformatically annotated as a $\beta$-xylosidase, designated Bgxg1, representing

121 the first study of a GH39-family enzyme from anaerobic fungi. Our results document the high

122 affinity, high specific activity, wide $\mathrm{pH}$ and temperature ranges, high thermal and $\mathrm{pH}$ stability of

123 this enzyme, and novel multiple activities.

124 Materials and Methods

125 Transcriptomics-guided selection of a GH39 enzyme for cloning and characterization.

126 As a part of an extensive transcriptomic analysis of lignocellulosic biomass degradation by the

127 anaerobic fungal isolate Orpinomyces sp. strain C1A (Couger et al. 2015), the most highly

128 transcribed gene annotated as a $\beta$-xylosidase was selected for cloning and biochemical

129 characterization. The selected m.21910 transcript (GenBank accession number KT997999) was

130 annotated as member of the GH39 CAZyme family based on the presence of the conserved

131 protein domain pfam01229 (Glyco_hydro_39) family. When strain C1A was grown on different

132 substrates (glucose, Corn Stover, Energy Cane, Switchgrass, and Sorghum), m.21910 constituted

$13358-84 \%$ of the transcriptional activity (i.e. normalized FPKM values) of all GH39 transcripts 
$134(\mathrm{n}=9)$, and $5.7-18.2 \%$ of the transcriptional activities of all C1A genes putatively annotated as $\beta$ -

135 xylosidases (members of GH39 and GH43, $\mathrm{n}=41$ ) (Couger et al. 2015). The gene encoding for

136 Bgxg1 protein was previously identified in the genome of strain C1A (GenBank contig accession

137 number ASRE01002650.1, range: 2346-3460, see GCA_000412615.1 for whole genome). The

$138 \operatorname{ctg} 7180000059688.1$ gene consists of $1115 \mathrm{bp}$ and no introns (refer to IMG gene ID 2518718918

139 for a visual representation of the gene, https://img.jgi.doe.gov/cgi-

$140 \mathrm{bin} / \mathrm{m} / \mathrm{main}$. cgi?section=TaxonDetail\&page=taxonDetail\&taxon_oid=2518645524). The protein

141 product is predicted to be extracellular and non-cellulosomal, based on the presence of a signal

142 peptide, and the absence of a CBM fungal dockerin domain, respectively.

143 Bgxgl sequence analysis and phylogeny.

144 To determine the phylogenetic affiliation of Bgxg1 and the overall topology and global 145 phylogeny of GH39 CAZymes, GH39 $\beta$-xylosidase sequences available in CAZY database 146 (http://www.cazy.org/GH39_all.html) (n=1145 total GH39 sequences, retrieved October 28,

1472015 , edited to remove $\alpha$-iduronidases and duplicates, resulting in $n=200 \beta$-xylosidases), in

148 addition to Bgxg1, were aligned using Clustal Omega (Sievers et al. 2011). The generated

149 alignment was used to construct a maximum likelihood tree in RAxML (Stamatakis 2014),

150 which was subsequently visualized and annotated using Mega6 (Sievers et al. 2011; Tamura et

151 al. 2013).

152 Synthesis, cloning, expression, and purification of Bgxgl protein.

153 bgxgl gene synthesis and cloning. A fraction (939 bp, positions 67-1035) of m.21910 transcript 154 was codon optimized for ideal expression in E. coli (see Fig. S1 for the alignment of the original 155 gene and codon-optimized gene), and the bgxgl insert was synthesized by a commercial provider 156 and inserted into a pET28a(+) plasmid (GenScript, Piscataway, NJ). The plasmid, pET28a(+)- 
157 bgxgl, harbors kanamycin resistance (kan) and NdeI and XhoI restriction sites for selection and

158 cloning. The pET28a(+)-bgxg1 plasmid was first transformed into One-Shot Chemically

159 Competent Top10 E. coli cells (Invitrogen, Carlsbad, CA), and the transformants were grown

160 overnight on LB-kanamycin agar $(15 \mu \mathrm{g} / \mathrm{mL})$ for selection. The purified plasmid was

161 electroporated into a protease-deficient BL21(DE3)pLysS E. coli strain (Novagen, EMD

162 Millipore, Darmstadt, Germany), possessing an additional chloramphenicol resistance $(\mathrm{cm})$

163 marker, using a single pulse of $1.8 \mathrm{kV}$ in $0.1 \mathrm{~cm}$ electrocuvettes. Transformants were grown on

164 LB agar using both kanamycin $(15 \mu \mathrm{g} / \mathrm{mL})$ and chloramphenicol $(34 \mu \mathrm{g} / \mathrm{mL})$ for selection and

165 screened for the presence of correctly sized inserts via colony PCR using T7 forward and reverse

166 primers.

167 Bgxgl expression and purification. Ten milliliters of overnight cultures of BL21(DE3)pLysS E.

168 coli cells transformed with pET28a(+)-bgxgl were used to inoculate 1 L LB broth, containing

169 kanamycin $(15 \mu \mathrm{g} / \mathrm{mL})$ and chloramphenicol $(34 \mu \mathrm{g} / \mathrm{mL})$. The culture was incubated at $37^{\circ} \mathrm{C}$

170 with shaking at $200 \mathrm{rpm}$ until an $\mathrm{OD}_{600}=0.6$ was reached. Isopropyl- $\beta$-D-thiogalactopyranoside

171 (IPTG, $1 \mathrm{mM}$ final concentration) was then added to induce protein production, and the culture

172 was gently shaken at room temperature overnight. Cells were then pelleted by centrifugation

$173\left(6,000 \times \mathrm{g}, 10\right.$ minutes, $\left.4^{\circ} \mathrm{C}\right)$ and the pellets were collected and stored at $-20^{\circ} \mathrm{C}$.

174 Preliminary small-scale experiments indicated that the protein is expressed in the

175 inclusion body fraction (Fig. S2). Inclusion body extraction was initiated by incubating the

176 cultures in B-Per Cell Lysis Reagent (Thermo Scientific, Grand Island, NY) (10 ml per $500 \mathrm{ml}$ of

177 culture) for 15 minutes at room temperature with gentle shaking to lyse the cells. The

178 homogenate was centrifuged $\left(10,000 \mathrm{x}\right.$ g, 30 minutes, $\left.4^{\circ} \mathrm{C}\right)$ and the inclusion body extraction

179 procedure (Grassick et al. 2004) was conducted on the cell pellet as follows: The pellet was 
180 resuspended in a urea-based inclusion body extraction buffer (20\% glycerol, $8 \mathrm{M}$ urea, $50 \mathrm{mM}$

181 sodium monobasic phosphate, $500 \mathrm{mM}$ sodium chloride, $\mathrm{pH}$ 8.0) for 30 minutes at room

182 temperature with gentle shaking. The homogenate was centrifuged $\left(10,000 \mathrm{x} \mathrm{g}, 30\right.$ minutes, $\left.4^{\circ} \mathrm{C}\right)$

183 and the resultant supernatant containing target inclusion body proteins was subsequently utilized

184 for refolding and purification procedures.

185 Recombinant protein refolding was achieved using slow dialysis as previously described

186 (Grassick et al. 2004). In brief, inclusion body extract was incubated with EDTA (1 mM final

187 concentration) and $\beta$-mercaptoethanol (100 $\mathrm{mM}$ final concentration) for 2 hours at room

188 temperature with gentle shaking, transferred to dialysis tubing (NMWL: 12,000 - 14,000 Da),

189 and placed for 3 hours into inclusion body exchange buffer (20\% glycerol, $8 \mathrm{M}$ urea, $50 \mathrm{mM}$

190 sodium monobasic phosphate, $500 \mathrm{mM}$ sodium chloride, $1 \mathrm{mM}$ EDTA, $\mathrm{pH}$ 8.0) for removal of

191 the $\beta$-mercaptoethanol. The buffer was refreshed and dialyzed for an additional 3 hours. The

192 dialysis tubing was then placed into a low-urea refolding buffer ( $2 \mathrm{M}$ urea, $50 \mathrm{mM}$ sodium

193 monobasic phosphate, $500 \mathrm{mM}$ sodium chloride, $1 \mathrm{mM}$ EDTA, $3 \mathrm{mM}$ reduced glutathione, 0.9

$194 \mathrm{mM}$ oxidized glutathione, $\mathrm{pH}$ 8.0) and dialyzed overnight, followed by a no-urea refolding buffer

195 (50 mM sodium monobasic phosphate, $500 \mathrm{mM}$ sodium chloride, $1 \mathrm{mM}$ EDTA, $3 \mathrm{mM}$ reduced

196 glutathione, $0.9 \mathrm{mM}$ oxidized glutathione, $\mathrm{pH} \mathrm{8.0)} \mathrm{for} 36$ hours.

197 Following dialysis, the contents of the tubing were centrifuged to remove insoluble,

198 precipitated proteins $\left(15,000 \mathrm{xg}, 15\right.$ minutes, $\left.4^{\circ} \mathrm{C}\right)$. The supernatant, containing refolded soluble

199 protein, was then exposed to a nickel-nitriloacetic acid (Ni-NTA, 1:1 ratio) slurry (UBPBio,

200 Aurora, CO), packed in a glass frit column ( 25 x 200 mm, 98 mL volume Kimble-Chase Kontes

201 Flex Column, Vineland, NJ), and allowed to incubate at $4^{\circ} \mathrm{C}$ for 1 hour on an orbital shaker.

202 Protein purification followed as detailed previously (Morrison et al. 2012). Samples were 
203 concentrated using Amicon Ultra-15 Centrifugal Filter Units (Millipore, NMWL $30 \mathrm{kDa}$ ) and

204 protein concentration was determined using a Qubit Fluorimeter (Thermo Scientific) in reference

205 to standard protein concentrations. Protein refolding was checked as activity against PNPX, as

206 described below. An SDS-PAGE gel was run to check protein size and purity, as previously

207 described (Laemmli 1970; Morrison et al. 2012).

208 Biochemical characterization of Bgxgl (Enzyme activity assays).

$209 \mathrm{pH}$ and temperature optima and stability. The $\mathrm{pH}$ range and subsequent $\mathrm{pH}$ optimum for Bgxg1

210 was determined by assaying its $\beta$-xylosidase activity (described below) at $\mathrm{pH} 3,4,5,6,7,8,9$,

211 and 10, using the following buffer systems: sodium acetate buffer ( $\mathrm{pH} 3.0-6.0)$, sodium

212 phosphate buffer ( $\mathrm{pH}$ 7.0-8.0), and glycine buffer ( $\mathrm{pH}$ 9.0-10). Similarly, the temperature range

213 and subsequent thermal optimum for Bgxg1 was determined by assaying its $\beta$-xylosidase activity

214 at $25,30,39,50$, and $60^{\circ} \mathrm{C}$. In a second and separate study, the stability of Bgxg1 after exposure

215 to $\mathrm{pH}$ extremes was determined by assaying its $\beta$-xylosidase activity following a one-hour

216 incubation at $\mathrm{pH} 3,4,5,6,7,8,9,10,11,12$, and 13 at $4^{\circ} \mathrm{C}$. The following $\mathrm{pH}$ buffering systems

217 were used for $\mathrm{pH}$ adjustment: sodium acetate buffer $(\mathrm{pH} 3.0-6.0)$, sodium phosphate buffer ( $\mathrm{pH}$

218 7.0-8.0), glycine buffer ( $\mathrm{pH}$ 9.0-10), sodium bicarbonate ( $\mathrm{pH} 11.0$ ), and $\mathrm{KCl}-\mathrm{NaOH}(\mathrm{pH} 12-13)$.

219 Similarly, in a separate study, the thermal stability of Bgxg1 was determined by assaying its $\beta$ -

220 xylosidase activity following a one-hour incubation at $4,25,30,37,39,50,60$, and $70^{\circ} \mathrm{C}$. In all

221 cases, $2.2 \mu \mathrm{g}$ of pure $\operatorname{Bgxg} 1$ was used, since this concentration was determined to be optimal in

222 initial testing. Following the one-hour long exposure at the above-described extremes, enzymatic

223 activity was tested at $39^{\circ} \mathrm{C}$ and $\mathrm{pH} 6.0$, the optimal conditions as determined for this enzyme. All

224 experiments were completed in triplicate, and relative specific activities in relation to the best

225 performing condition (100\% activity) were reported. 
226 Enzyme activity assays. All enzyme assays with Bgxg1 were conducted in $\mathrm{pH} 6.0$ buffer and at

$22739^{\circ} \mathrm{C}$, as these conditions were determined to be optimal for Bgxg1. All reagents were purchased

228 from Sigma Aldrich (St. Louis, MO) unless noted otherwise.

229 Endoglucanase, exoglucanase, xylanase, and mannanase activities were determined using

230 a DNS (3,5-dinitrosalicyclic acid)-based assay (Breuil \& Saddler 1985), with carboxymethyl

231 cellulose sodium salt (CMC, $1.25 \% \mathrm{w} / \mathrm{v})$, avicel microcrystalline cellulose $(1.25 \% \mathrm{w} / \mathrm{v})$,

232 beechwood xylan $(1.25 \% \mathrm{w} / \mathrm{v})$, and locust bean gum $(0.5 \% \mathrm{w} / \mathrm{v})$ as substrates, respectively.

233 Glucose, xylose, or mannose were utilized for the generation of a standard curve, dependent on

234 the substrate being tested.

235 Cellobiohydrolase, $\beta$-xylosidase, arabinosidase, mannosidase, $\beta$-glucosidase, $\beta$ -

236 galactosidase, and acetyl xylan esterase activities were determined using $(10 \mathrm{mM})$ of the $p$ -

237 nitrophenol-based (PNP) substrates: $p$-nitrophenyl- $\beta$-D-cellobioside (PNPC,), $p$-nitrophenyl- $\beta$-D-

238 xylopyranoside (PNPX), $p$-nitrophenyl- $\beta$-D-arabinofuranoside (PNPA) $p$-nitrophenyl- $\beta$-D-

239 mannoside (PNPM), $p$-nitrophenyl- $\beta$-D-glucopyranoside (PNPG), $p$-nitrophenyl- $\beta$-D-

240 galactopyranoside (PNPGal), and $p$-nitrophenyl-acetate (PNPAc), respectively (Dashtban et al.

241 2010; Kubicek 1982; Zhang et al. 2009). Assays were conducted in sodium acetate buffer with

242 sodium carbonate $(1 \mathrm{M})$ as a stop reagent. The release of PNP was measured colorimetrically at

$243420 \mathrm{~nm}$, following the addition of the stop solution. $\alpha$-glucuronidase activity was assayed using

244 the Megazyme $\alpha$-glucuronidase assay kit (Wicklow, Ireland).

245 All experiments were conducted in triplicate. One unit of enzymatic activity (U) was

246 defined as one $\mu$ mol of products (reducing sugar equivalents in DNS assays, PNP released in

247 PNP substrate-based assays, and aldouronic acid in $\alpha$-glucuronidase assay) released from the 
248 substrate per minute. Specific activity was calculated by determining the units released per mg of

249 enzyme.

250 Enzyme kinetics. Standard procedures were used to determine the $K_{m}, V_{\max }$, and specific activity

251 of Bgxg1 on all substrates described above (Lineweaver \& Burk 1934). $K_{m}$ and $V_{\max }$ values were

252 obtained using double-reciprocal Lineweaver-Burke plots, which were used to extrapolate from

253 experimentally-derived values using a constant protein concentration $(2.2 \mu \mathrm{g})$ and variable PNP-

254 based substrate concentration $(0.1-100 \mathrm{mM})$ (Lineweaver \& Burk 1934). Given the extinction

255 coefficient of p-nitrophenol (PNP) is $17 / \mathrm{mM} / \mathrm{cm}$ at $400 \mathrm{~nm}$ (Bessey \& Love 1952), for a $1 \mathrm{~cm}$

256 path length cuvette and absorbance minimum of 0.010 , reliable $K_{m}$ detection limits in such PNP-

257 based spectrophotometric assays is $\approx 500 \mathrm{nM}$. Therefore, $K_{m}$ values $<500 \mathrm{nM}$ are referred to as

258 BDL (below detection limit).

259 Substrate competition assays. Competitive inhibition experiments were conducted to determine

260 whether the observed multiple oligosaccharide hydrolase activities are catalyzed via a single or

261 multiple active sites. In such experiments, the effect of cellobiose (as a competitive inhibitor) on

262 the $\beta$-xylosidase activity of Bgxg1 was measured by conducting the $\beta$-xylosidase assay, using 10

$263 \mathrm{mM}$ of PNPX as the substrate, in the presence of different concentrations of cellobiose $(0,10$,

264 and $20 \mathrm{mM}$ ) and evaluating the impact of cellobiose presence on the release of PNP. Conversely,

265 the effect of xylobiose (as a competitive inhibitor) on the $\beta$-glucosidase activity of Bgxg1 was

266 measured by conducting the $\beta$-glucosidase assay (using $10 \mathrm{mM}$ of PNPG as the substrate) in the

267 presence of different concentrations of xylobiose $(0,10$, and $20 \mathrm{mM})$, and evaluating the impact

268 of xylobiose presence on the release of PNP. In both experiments, the effect of inhibitor

269 concentration on $K_{m}$ and $V_{\max }$ was evaluated using Lineweaver-Burke plots (Lineweaver \& Burk

270 1934). All experiments were conducted in triplicate. 
271 Substrate preferences of Bgxg1 were determined by conducting a substrate competition

272 assay, where $\operatorname{Bgxg} 1$ (2.2 $\mu \mathrm{g}$ of pure enzyme preparation) was challenged by a mixture of

273 xylobiose $(10 \mathrm{mM})$ and cellobiose $(10 \mathrm{mM})$. The kinetics of xylose and glucose release were

274 compared to the results obtained in control experiments where only one substrate (xylobiose or

275 cellobiose) was utilized. Samples were taken at 0, 1, 5, 10, 15, 30, and 60 minutes for the

276 determination of the glucose and xylose concentrations. Glucose was assayed using PGO

277 Enzyme Preparation Capsules (Sigma-Aldrich, St. Louis, MO) and xylose was assayed using

278 Megazyme Xylose Kit (Wicklow, Ireland). All experiments were conducted in triplicate.

279 Bgxg1 modeling.

280 Homology modeling by Iterative Threading ASSEmbly Refinement (I-TASSER) (Roy et al.

281 2010; Yang et al. 2015; Zhang 2008), was conducted to generate a three-dimensional model of

282 Bgxg1 using Thermoanaerobacterium saccharolyticum $\beta$-xylosidase (PBD entry 1UHV) as a

283 template. PyMOL was used to align the Bgxg1 structural prediction to that of

284 Thermoanaerobacterium saccharolyticum (PBD entry 1UHV) to examine and speculate the

285 impact of variations in amino acids residue on the enzyme's active site topology and putative

286 substrate binding capacities (PyMol).

\section{Results}

288 Bgxg1 phylogenetic affiliation.

289 Phylogenetic analysis grouped all GH39 sequences into 4 phylogenetically-resolved and

290 bootstrap-supported clades (Classes I-IV in Fig. 1). Orpinomyces sp. strain C1A Bgxg1 protein

291 belonged to Class III, forming a well-supported cluster with GH39 proteins from the anaerobic

292 fungus Piromyces sp. strain E2, as well as GH39 proteins from the bacterial genera Clostridium 293 and Teredinibacter (70-74\% sequence identities) (Fig. 1). To our knowledge, none of the GH39 
294 proteins within this specific cluster, or in the entire Class III GH39, has been biochemically

295 characterized.

296 Physiological characterization.

297 SDS-PAGE results show that the Bgxg1 protein is consistent with the predicted size of $42.7 \mathrm{kDa}$ 298 (protein predicted molecular weight is $39.6 \mathrm{KDa}+0.996 \mathrm{kDa}$ linker $+2.101 \mathrm{kDa}$ double 299 histidine tag) (Fig. S3).

300 The thermal and $\mathrm{pH}$ ranges and optima were determined by conducting assays at a range 301 of temperatures and $\mathrm{pH}$ 's, as described above. Bgxg1 exhibited activity in a wide range of $\mathrm{pH}$ (3302 8) and temperatures $\left(25-60^{\circ} \mathrm{C}\right)$, with optimal activity at $\mathrm{pH} 6$ and $39^{\circ} \mathrm{C}$ (Fig. 2A, 2B). The

303 thermal and $\mathrm{pH}$ stabilities of Bgxg1 were examined by conducting activity assays post-stress ( $\mathrm{pH}$ 304 or thermal)-incubations as described above. Bgxg1 retained more than $80 \%$ of its specific 305 activity post-application of pH stress ranging between 6 and 11 (Fig. 2C), and $60 \%$ of its specific 306 activity post application of $\mathrm{pH}$ stress of 4,5 , and 12 (Fig. 2C). Further, Bgxg1 retained $\geq 70 \%$ of 307 its specific activity across the broad range of temperature stressors applied $\left(4-70^{\circ} \mathrm{C}\right)$ (Fig. 2D). 308 In addition, exposure to $\mathrm{pH}$ stress from 6-11 and temperature stress from $4-70{ }^{\circ} \mathrm{C}$ did not 309 produce results that were significantly different from the optimal conditions ( $p$-value $>0.05,95 \%$ 310 confidence interval, Fig. 2).

\section{Substrate specificities and kinetics.}

312 To date, all characterized GH39 enzymes exhibit a narrow substrate range ( $\beta$-xylosidase or $\alpha$-L313 iduronidase) (Table S1). As predicted by sequence analysis, Bgxg1 exhibited $\beta$-xylosidase 314 activity (11.5 $\pm 1.2 \mathrm{U} / \mathrm{mg}$, Table 1$)$, strong as compared to previously reported $\beta$-xylosidase 315 activities (Table S1). While having a $\beta$-xylosidase activity greater than the majority of the field 316 in Table S1, Bgxg1 does have lower $\beta$-xylosidase activity than other anaerobic fungi 
317 Neocallimastix frontalis (16 U/mg, (Hebraud \& Fevre 1990)), Neocallimastix patriciarum (30.4

318 U/mg, (Zhu et al. 1994)), Piromyces communis (28 U/mg, (Hebraud \& Fevre 1988)), and

319 Sphaeomonas communis (27 U/mg, (Hebraud \& Fevre 1988) (Table 1, S1), however, these are

320 not of the same GH-family. Interestingly, in addition to $\beta$-xylosidase activity, Bgxg1 also

321 exhibited strong $\beta$-glucosidase $(73.4 \pm 7.15 \mathrm{U} / \mathrm{mg}), \beta$-galactosidase $(54.6 \pm 2.26 \mathrm{U} / \mathrm{mg})$, and

322 weak xylanase $(10.8 \pm 1.25 \mathrm{U} / \mathrm{mg}$ ) activities (Table 1$)$, as compared to reported activities from

323 previously characterized enzymes (Tables S2-S4). Our extensive literature review identified 63

324 enzymes that have been biochemically-characterized to have $\beta$-glucosidase activity and, of these,

325 only seven have a reported specific activity higher than that of Bgxg1 (Table S2), including

326 Candida peltata (108 U/mg, (Saha \& Bothast 1996)), Thermoascus aurantiacus (232 U/mg,

327 (Tong et al. 1980)), and Trichoderma reesei (768 U/mg, (Takashima et al. 1998)). Similarly, we

328 only identified three $\beta$-galactosidase with a reported higher activity than Bgxg1 (Table S3),

329 including Alicyclobacillus acidocaldarius (229 U/mg, (Yuan et al. 2008)), Bifidobacterium

330 adolescentis (526 U/mg, (Hinz et al. 2004)), and Thermus aquaticus (1750 U/mg, (Ulrich et al.

331 1972)). On the other hand, the xylanase activity of Bgxg1 is relatively weak, with many

332 previously reported xylanases exhibiting a much higher specific activity (Table S4), such as

333 Aspergillus niger (19 U/mg, $25 \mathrm{U} / \mathrm{mg}, 35 \mathrm{U} / \mathrm{mg}$, and $48 \mathrm{U} / \mathrm{mg}$, (John et al. 1979)) and

334 Trichoderma reesei (46 U/mg, (Takashima et al. 1998)). Bgxg1 exhibited no detectable

335 exoglucanase, endoglucanase, mannanase, arabinosidase, acetyl xylan esterase,

336 cellobiohydrolase, mannosidase, or $\alpha$-glucuronidase activities.

337 In addition to its high $\beta$-xylosidase, $\beta$-glucosidase, and $\beta$-galactosidase specific activities,

338 Bgxg1 exhibited remarkably high affinities towards all examined substrates, with $K_{m}$ values 
339 (calculated via extrapolation through Lineweaver-Burke plot) in the low nM range for PNPG and

340 PNPGal, the low $\mu \mathrm{M}$ range for PNPX (Table 2, Table S1-S4).

341 Substrate competition studies.

342 Substrate competition studies were conducted using a variable concentration of an unlabeled

343 substrate (acting as an inhibitor) and a fixed concentration of a chromophore (PNP-based)

344 substrate (Table 3). The results strongly suggest the occurrence of cross-substrate competitive

345 inhibition between xylobiose and cellobiose (Table 3), since the presence of increasing

346 concentrations of a single substrate lowers the specific activity and increases the $K_{m}$ of the

347 enzyme towards the other substrate, whilst not affecting its $V_{\max }\left(K_{m}\right.$ and $V_{\max }$ calculated via

348 extrapolation through Lineweaver-Burke plot). This pattern strongly indicates that a single active

349 site is responsible for the observed activities (Table 3), a conclusion that is in agreement with the 350 lack of identifiable additional domains other than pfam01229 in Bgxg1, as well as with the 351 structural modeling data described below.

352 In single substrate assays, Bgxg1 was capable of converting cellobiose to glucose and

353 xylobiose to xylose at a very fast rate (Fig. 3A, 3B). This reaction occurs more quickly for

354 xylobiose, as a stable maximal xylose concentration is reached after only 1 minute of incubation

355 (Fig. 3B), compared to 15 minutes for glucose release from cellobiose (Fig. 3A). However, the

356 extent of sugar release at the conclusion of the experiment was higher in cellobiose incubations

357 (Fig. 3A) than xylobiose incubations (Fig. 3B). Competition studies using equimolar

358 concentrations of both substrates revealed the preference of Bgxg1 for xylobiose, since a higher

359 proportion of xylose rather than glucose was detected within the first 15 minutes of the

360 incubation (Fig. 3C). Nevertheless, the final concentrations of sugars released after 60 minutes of

361 incubation did not differ when comparing single substrate versus competition experiments (Fig. 
362 3A-3C). Similar to the patterns observed in single substrate assays, Bgxg1 reduced a larger

363 amount of cellobiose to glucose than xylobiose to xylose in competition experiments (Fig. 3C),

364 which is consistent with the higher affinity (lower $K_{m}$ value) of Bgxg1 for PNPG $(12.5 \mathrm{nM})$ over

$365 \operatorname{PNPX}(4.85 \mu \mathrm{M})($ Table 2$)$.

366 Structure activity predictions.

367 The Bgxg1 protein sequence was submitted to I-TASSER for structural prediction by Iterative

368 Threading ASSEmbly Refinement (Roy et al. 2010; Yang et al. 2015; Zhang 2008) utilizing the

$369 \beta$-xylosidase originating from Thermoanaerobacterium saccharolyticum (PBD entry 1UHV) as a

370 template for model creation (Roy et al. 2010; Yang et al. 2004; Yang et al. 2015; Zhang 2008).

371 Bgxg1 is predicted to have three distinct domains: a catalytic $(\alpha / \beta)_{8}$ barrel fold domain (position

372 26-307), a small $\alpha$-helical domain (position 1-25), and a $\beta$ sandwich domain (position 308-344)

373 (Fig. S4). Overall, the structure is predicted to contain $11 \beta$-sheets $\left(8\right.$ in $(\alpha / \beta)_{8}$-barrel, 3 in $\beta$ -

374 sandwich), and $10 \alpha$-helices (8 in $(\alpha / \beta)_{8}$-barrel, 2 in $\alpha$-domain). The catalytic $(\alpha / \beta)_{8}$-barrel fold

375 domain is predicted to consist of eight parallel $\beta$-sheets $(\beta 1-\beta 8)$, and eight parallel $\alpha$-helices $(\alpha 1$ -

$376 \alpha 8$ ). Consistent with $\beta$-xylosidases of Thermoanaerobacterium saccharolyticum (1UHV) and

377 Geobacillus stearothermophilus (1PX8), the active site pocket of Bgxg1 is predicted to be

378 located on the upper side of the $(\alpha / \beta)_{8}$-barrel (Czjzek et al. 2005; Yang et al. 2004) (Fig. S4).

379 Alignment and structural predictions identified the conservation of the general acid-base active

380 site residue Glu127 in the C-terminal of $\beta 3$, as part of the GH39-conserved Asn126-Glu127-

381 Pro128 motif as well as the nucleophilic residue Glu225 in $\beta 6$ (Fig. 4) (Czjzek et al. 2005; Yang

382 et al. 2004).

383 Using the predicted model we sought to infer structural differences potentially

384 responsible for the observed relaxed substrate specificities in Bgxg1 by investigating the amino 
385 acid conservation patterns between Bgxg1 and all structurally and/or biochemically-

386 characterized $\beta$-xylosidases. These enzymes are: Thermoanaerobacterium saccharolyticum $\beta$ -

387 xylosidase (Yang et al. 2004), Geobacillus stearothermophilus $\beta$-xylosidase (Bhalla et al. 2014;

388 Czjzek et al. 2005), and Bacillus halodurans C-125 protein BH1068 (Wagschal et al. 2008), all

389 of which belong to Class II (Fig. 1), as well as Caulobacter crescentus CcXynB2 (Correa et al.

390 2012), which belongs to Class I (Fig. 1). All of these enzymes have previously been reported to

391 possess $\beta$-xylosidase activity (Bhalla et al. 2014; Correa et al. 2012; Czjzek et al. 2005;

392 Wagschal et al. 2008; Yang et al. 2004). We focused on 25 amino acids in two groups: (i) those

393 previously shown to be important for $\beta$-xylosidase activity (Czjzek et al. 2005; Yang et al. 2004)

394 [this group includes (in addition to the conserved general acid-base and nucleophilic active sites

395 described above) amino acids providing the tight hydrogen bonding necessary to stabilize the 396 xylosyl-enzyme intermediate formed during the reaction, such as Arg52, His54, Asn159, His228, 397 Tyr230, Glu278, Trp315, Glu322, and Glu323 (locations refer to position in 1UHV)], as well as 398 (ii) those physically interacting with the active site as deduced by the predicted Bgxg1 model 399 (Fig. S3A) [this group includes Val46, Va181, Ile124, Trp125, Gly130, Thr131, Trp132, Phe139, 400 Pro162, Cys163, Tyr164, Ser165, Lys171, His192, Asn242, and Lys247 (locations refer to 401 position in Bgxg1)]. Of these 25 amino acids, 15 differed between Bgxg1 and the four other 402 proteins. Five of these 15 amino acids were not conserved amongst any of the five sequences 403 studied and so were not further investigated (Fig. 4). Therefore, 10 distinct differences (8 404 substitutions and 2 deletions) between Bgxg1 on one hand and the four biochemically405 characterized $\beta$-xylosidases on the other were identified (Table 4). These differences that are 406 predicted to exist in or around the active site of Bgxg1 would putatively impact the size, charge, 407 and/or polarity within the active site (Table 4, Fig. S4). 
409 Bgxg1, or it could be specific to all GH39 CAZymes of anaerobic fungi (e.g. Class III-C), or to

410 the entire Class III $\beta$-xylosidases. Based on the above speculations about the amino acids

411 potentially responsible for Bgxg1 relaxed specificity, we further investigated the conservation of

412 these 10 amino acid changes (Table 4) within class III of GH39 proteins. Bgxg1 (as well as other

413 GH39 proteins encoded in C1A genome), all three GH39 proteins from the Piromyces genome

414 (accession numbers shown in Fig. 1), and all additional sequences from Class III-C belonging to

415 the genera Clostridium and Teredinibacter were found to encode 9 of the 10 observed amino

416 acid substitutions (Table 4). However, within the broader Class III, little similarity in key amino

417 acids was observed between Bgxg1 sequences and $\beta$-xylosidases belonging to Class III-A, III-B,

418 or III-D (Table 4). Collectively, these results putatively suggest that the observed relaxed

419 specificity in Bgxg1 could be exclusive to Class III-C $\beta$-xylosidases.

\section{Discussion}

421 In this study, we used a transcriptomics-guided approach to identify, clone, express, and

422 characterize a GH39 protein (Bgxg1) from the anaerobic gut fungus Orpinomyces sp. strain

$423 \mathrm{C} 1 \mathrm{~A}$. Our results demonstrate that the expressed protein is multifunctional, possessing strong $\beta$ 424 xylosidase (11.5 U/mg), $\beta$-glucosidase (73.4 U/mg), and $\beta$-galactosidase $(54.6 \mathrm{U} / \mathrm{mg})$ activities, 425 as well as a weak xylanase activity $(10.8 \mathrm{U} / \mathrm{mg})$ (Table 1,2$)$, as compared to previously 426 characterized enzymes (Tables S1-S4). This novel multi-functionality has not been previously 427 reported in GH39 enzymes (Bhalla et al. 2014), and therefore this work expands on the known 428 activities of GH39 CAZyme family. Further, Bgxg1 retains high levels of activity over a wide 429 range of temperatures $\left(>80 \%\right.$ of activity retained between $\left.4-70^{\circ} \mathrm{C}\right)$ (Fig. $\left.2 \mathrm{D}\right)$ and $\mathrm{pH}$ values 430 (>80\% of activity retained between $\mathrm{pH} 6-11)$ (Fig. 2C). Though the composition of commercial 
431 enzymes cocktails are largely proprietary, the presence of 80-200 different components within a

432 mixture has been previously reported (Banerjee et al. 2010; Van Dyk \& Pletschke 2012). It is

433 intuitive to think that the inclusion of such a large number of enzymes represents a large

434 contribution to the cost of production. It is here that Bgxg1 would be beneficial, as the inclusion

435 of a single enzyme, possessing multiple strong activities, would lower the cost of production in

436 biorefineries and therefore would be beneficial to the bottom line.

437 In addition to its relaxed substrate specificity, the enzyme displays strong kinetic

438 properties (high specific activity and affinity) towards its multiple substrates ( $K_{m}$ and $V_{\max }$ values

439 calculated via extrapolation through Lineweaver-Burke plot). As a $\beta$-xylosidase, Bgxg1 has one

440 of the highest $\beta$-xylosidase specific activity among all reported ambient $\left(<50^{\circ} \mathrm{C}\right) \beta$-xylosidases,

441 but lower than other anaerobic fungi Neocallimastix frontalis (16 U/mg, (Hebraud \& Fevre

442 1990)), Neocallimastix patriciarum (30.4 U/mg, (Zhu et al. 1994)), Piromyces communis (28

$443 \mathrm{U} / \mathrm{mg}$, (Hebraud \& Fevre 1988)), and Sphaeomonas communis (27 U/mg, (Hebraud \& Fevre

444 1988) (Table 1, S1). Bgxg1 also one of the highest specific activities amongst known GH39 $\beta$ -

445 xylosidases (Tables 1, S1), with a lower specific activity than the thermophilic

446 Thermoanaerobacterium saccharolyticum (53.8 U/mg, (Shao et al. 2011)) and the thermophilic

447 Geobacillus stearothermophilus (133 U/mg, (Bhalla et al. 2014)). Compared to other

448 characterized $\beta$-glucosidases, Bgxg1 has the highest specific activity for all ambient temperature

$449 \beta$-glucosidases, and one of the highest reported specific activities among all $\beta$-glucosidase

450 (members of GH1, GH3, GH5, GH9, and GH30 (Cairns \& Esen 2010)), regardless of optimal

451 temperature and GH affiliation (Tables 1, S2). Finally, compared to other characterized $\beta$ -

452 galactosidases, Bgxg1 has the highest specific activity for all ambient temperature $\beta$ -

453 galactosidases, and one of the highest reported specific activities among all $\beta$-galactosidases 
454 (members of GH1, GH2, GH35, and GH42 (Skalova et al. 2005)) regardless of optimal

455 temperature and GH affiliation (Tables 1, 2, S4).

456

We reason that the observed kinetics and substrate specificity of Bgxg1 are beneficial for

457 strain C1A and are highly desirable for a saccharolytic enzyme acting within the highly

458 competitive rumen environment, where strain C1A originally existed (Orpinomyces sp. strain

459 C1A was isolated from the feces of an angus steer (Youssef et al. 2013)). The high specific

460 activity and high substrate affinity may aid in fast and efficient scavenging of sugars from the

461 surrounding environment, where competition for sugars/oligosaccharide produced by

462 saccharolytic enzymes are intense, and where free sugar levels are permanently low (Garcia-

463 Vallve et al. 2000). We hence speculate that the survival in an anaerobic, eutrophic, and highly

464 competitive environment might be responsible for the acquisition, retention and directed

465 evolution of anaerobic fungal $\beta$-xylosidases towards superior kinetics and relaxed specificities.

466 Sequence analysis and structural predictive modeling (Figs. 4 and S4), and substrate

467 competition experiments (Table 3) predict the presence of a single conserved active site within

468 the $(\alpha / \beta)_{8}$-barrel fold structure typically observed in GH39-family enzymes (Czjzek et al. 2005;

469 Yang et al. 2004) (with the conserved catalytic nucleophile (Glu225) and general acid-base

470 residue (Glu127)) and potentially mediating all observed hydrolytic activities). To provide clues

471 regarding the structural basis of the observed multi-functionality, comparison of amino acid

472 conservation patterns putatively affecting the active site topology between Bgxg1 and

473 biochemically characterized GH39 xylosidases, all four of which display no additional activities

474 beyond $\beta$-xylosidase, was undertaken. We identified ten different distinct amino acid changes (8

475 substitutions and 2 deletions) (Table 4, Figs. S4 and 5) in Bgxg1 that putatively affect the

476 polarity (Tyr vs. Val46, Phe vs. Thr131, Tyr vs. Phe139, Ala vs. Cys163, Trp vs. Lys171, Tyr vs. 
477 Leu194, and Ala vs. Arg242), constitute significant size changes (Tyr vs. Val46, Phe vs. Thr131,

478 Tyr vs. Leu194, and Ala vs. Arg242), result in the addition of charged moieties or unique

479 functional groups (Asn vs. Asp129, Ala vs. Cys163, Trp vs. Lys171, and Ala vs. Arg242), or

480 result in the deletion of a negatively charged residue, previously determined to be important

481 (Glu322-323 vs. deletion) to the active site (Czjzek et al. 2005). The impact of these speculated

482 changes is unclear, and it remains to be seen if any, all, or a combination of the above differences

483 is responsible for the observed relaxed specificity. However, while all these amino acid changes

484 are speculated to theoretically explain the relaxed specificity of Bgxg1, one such difference is

485 peculiar and deserves special scrutiny; deletions/gaps in the Bgxg1 sequence as opposed to

486 negatively charged glutamic acids in the other four sequences (Table 4, Fig. S4S). GH39

487 enzymes belong to the wider family of $\beta$-1,4-retaining hydrolases of clan GH-A e.g. GH1 $\beta$ -

488 glucosidase and GH5 cellulases. Differences in structure between $\beta$ 1,4-glucose cleaving

489 enzymes and $\beta$ 1,4-xylose cleaving enzymes within clan GH-A have been extensively

490 investigated (Czjzek et al. 2005; Czjzek et al. 2001; Ducros et al. 1995; Hovel et al. 2003;

491 Verdoucq et al. 2004). Such studies have demonstrated that, within the active site of $\beta$ 1,4-

492 glucose cleaving enzymes, a Gln residue (corresponding to position 39 in the enzyme dhurinase

493 of Sorghum bicolor (Czjzek et al. 2005; Ducros et al. 1995; Verdoucq et al. 2004)) interacts with

494 the substrate by forming a hydrogen bond with O3 and O4 of the glucose moiety (Czjzek et al.

495 2005; Ducros et al. 1995). On the other hand, $\beta$ 1,4-xylosidases acting on C5 sugar dimers

496 contain a Glu residue in lieu of Gln (at position 322-323 in Thermoanaerobacterium

497 saccharolyticum, Fig. 4 and S4, Table 4) that binds to O3 and O4 of the xylose moiety (Czjzek et

498 al. 2005). Interestingly, these Glu residues are aligned with a gap in the sequence of the

499 multifunctional Bgxg1 (Fig. 4), with no apparent occurrence of either Glu or Gln amino acids 
500 within the vicinity. Structurally predictive modeling suggests that in lieu of these Glu322-323

501 residues (1UHV numbering) Bgxg1 is predicted to possess Gly-Arg at an approximately

502 sterically-similar location near the active site (Figure S4R-S), representing a significant change

503 from two negatively-charged residues, to an uncharged and positively-charged pair of residues.

504 Since the Glu residues in biochemically characterized $\beta$-xylosidases are shown to be important

505 for stabilizing intermediates (Czjzek et al. 2005), the predicted absence of these residues in

506 Bgxg1 and their speculated replacement with Gly-Arg suggests that Bgxg1 might employ a

507 different mechanism for stabilizing its intermediates during the catalytic process; however, this

508 speculation will require further investigation.

509 The ecological relevance, global distribution, and evolutionary patterns of multi-

510 functionality within GH39 $\beta$-xylosidases remain to be conclusively determined. Phylogenetic

511 analysis demonstrated the occurrence of nine out of ten amino acids substitutions/deletions in all

512 sequenced members of Class III-C, residues which we speculate to be of importance to the

513 observed multi-functionality of Bgxg1, but as Bgxg1 is the only biochemically-characterized

514 enzyme within Class III, this analysis is purely speculative (Table 4, alignment in Fig. S5). In

515 addition to anaerobic fungal sequences, Class III-C $\beta$-xylosidases contain sequences from the

516 genera Clostridium and Teredinibacter (Fig. 1). Since it has been previously demonstrated that

517 the xylanolytic machinery in anaerobic fungi, including $\beta$-xylosidases, has been acquired from

518 bacteria via horizontal gene transfer (Youssef et al. 2013), and speculating that some or all of the

519 amino acids substitutions/deletions in members of class III-C collectively account for the

520 observed multi-functionality (though it is unknown, at this time, whether these GH39 enzymes

521 possess this multi-functionality), therefore we reason that the observed distribution pattern

522 suggests the evolution of relaxed specificity in GH39 $\beta$-xylosidases within the domain Bacteria, 
523 prior to the acquisition of GH39 $\beta$-xylosidases by the anaerobic fungi and that the acquired

524 capability is speculated to be retained in all anaerobic fungal GH39 $\beta$-xylosidases.

\section{Conclusions}

526 In conclusion, we have characterized a novel $\beta$-xylosidase that represents the first GH39-family

527 enzyme cloned and expressed from anaerobic fungi. The enzyme is multi-functional, capable of

528 hydrolyzing cellobiose, xylobiose, as well as several PNP-glycosides. It also displays high

529 affinity towards various substrates, retains activity over a wide range of temperatures and $\mathrm{pHs,}$

530 and possesses excellent temperature and thermal stability. Structurally predictive modeling

531 identified putative differences which potentially could account for the observed relaxed

532 specificity. Collectively, these capabilities render Bgxg1 an excellent candidate for inclusion in

533 enzyme cocktails mediating cellulose and hemicellulose saccharification from lignocellulosic

534 biomass (Morrison et al. 2016).

\section{Acknowledgements}

536 We thank Dr. Gilbert John (Oklahoma State University) for supplying the E. coli

537 BL21(DE3)pLysS cells used in this study. We also thank Dr. Robert Gruninger for helpful 538 discussions. 
541 Banerjee G, Scott-Craig JS, and Walton JD. 2010. Improving Enzymes for Biomass Conversion: A Basic Research Perspective. Bioenergy Research 3:82-92. 10.1007/s12155-009-9067-5

543 Bessey OA, and Love RH. 1952. Preparation and measurement of the purity of the phosphatase 544 reagent, disodium para-nitrophenyl phosphate. J Biol Chem 196:175-178.

545 Bhalla A, Bischoff KM, and Sani RK. 2014. Highly thermostable GH39 beta-xylosidase from a Geobacillus sp strain WSUCF1. BMC Biotechnol 14:963-973. Artn 963 10.1186/S12896014-0106-8

548

549

550

551

552

553

554

555

556

557

558

559

560

561

562

Borneman WS, Akin DE, and Ljungdahl LG. 1989. Fermentation products and plant-cell walldegrading enzymes produced by monocentric and polycentric anaerobic ruminal fungi. Appl Environ Microbiol 55:1066-1073.

Brennan Y, Callen WN, Christoffersen L, Dupree P, Goubet F, Healey S, Hernandez M, Keller M, Li K, Palackal N, Sittenfeld A, Tamayo G, Wells S, Hazlewood GP, Mathur EJ, Short JM, Robertson DE, and Steer BA. 2004. Unusual microbial xylanases from insect guts. Appl Environ Microbiol 70:3609-3617. Doi 10.1128/Aem.70.6.3609-3617.2004

Breuil C, and Saddler JN. 1985. Comparison of the 3,5-dinitrosalicylic acid and Nelson-Somogyi methods of assaying for reducing sugars and determining cellulase activity. Enzyme Microb Tech 7:327-332. Doi 10.1016/0141-0229(85)90111-5

Bronnenmeier K, and Staudenbauer WL. 1988. Purification and properties of an extracellular beta-glucosidase from the cellulolytic thermophile Clostridium stercorarium. Appl Microbiol Biot 28:380-386.

Cairns JRK, and Esen A. 2010. Beta-glucosidases. Cell Mol Life Sci 67:3389-3405. $10.1007 / \mathrm{s} 00018-010-0399-2$ 
563 Correa JM, Graciano L, Abrahao J, Loth EA, Gandra RF, Kadowaki MK, Henn C, and Simao

564 RDG. 2012. Expression and characterization of a GH39 beta-xylosidase II from

565

566 Caulobacter crescentus. Appl Biochem Biotech 168:2218-2229. 10.1007/s12010-012-

567

568 9931-1

569 isolate Orpinomyces sp. strain C1A. Biotechnol Biofuels 8:208-224.

570

Czjzek M, Ben David A, Braman T, Shoham G, Henrissat B, and Shoham Y. 2005. Enzyme-

571 substrate complex structures of a GH39 beta-xylosidase from Geobacillus

572 stearothermophilus. J Mol Biol 353:838-846. 10.1016/j.jmb.2005.09.003

573 Czjzek M, Cicek M, Zamboni V, Burmeister WP, Bevan DR, Henrissat B, and Esen A. 2001. 574 Crystal structure of a monocotyledon (maize ZMGlu1) beta-glucosidase and a model of

575

576 its complex with p-nitrophenyl beta-D-thioglucoside. Biochem J 354:37-46. Doi 10.1042/0264-6021:3540037

577 Dashtban M, Maki M, Leung KT, Mao CQ, and Qin WS. 2010. Cellulase activities in biomass 578 conversion: measurement methods and comparison. Crit Rev Biotechnol 30:302-309.

579 $10.3109 / 07388551.2010 .490938$

580

581 1995. Crystal structure of the catalytic domain of a bacterial cellulase belonging to family

582 5. Structure 3:939-949. Doi 10.1016/S0969-2126(01)00228-3

583 Elshahed MS. 2010. Microbiological aspects of biofuel production: current status and future directions. $J$ Adv Res 1:103-111. 
585 Garcia-Vallve S, Romeu A, and Palau J. 2000. Horizontal gene transfer of glycosyl hydrolases of 586 the rumen fungi. Mol Biol Evol 17:352-361.

587 Grassick A, Murray PG, Thompson R, Collins CM, Byrnes L, Birrane G, Higgins TM, and 588 Tuohy MG. 2004. Three-dimensional structure of a thermostable native 589 cellobiohydrolase, CBHIB, and molecular characterization of the cel7 gene from the

590

591 filamentous fungus, Talaromyces emersonii. Eur J Biochem 271:4495-4506.

592 593

Harhangi HR, Freelove AC, Ubhayasekera W, van Dinther M, Steenbakkers PJ, Akhmanova A, $10.1111 / \mathrm{j} .1432-1033.2004 .04409 . \mathrm{x}$

594 van der Drift C, Jetten MS, Mowbray SL, Gilbert HJ, and Op den Camp HJ. 2003. Ce16A, a major exoglucanase from the cellulosome of the anaerobic fungi Piromyces sp. 595 E2 and Piromyces equi. Biochim Biophys Acta 1628:30-39.

596

Hebraud M, and Fevre M. 1988. Characterization of glycoside and polysaccharide hydrolases

597

598 secreted by the rumen anaerobic fungi Neocallimastix frontalis, Sphaeromonas communis and Piromonas communis. J Gen Microbiol 134:1123-1129.

599

600

Hebraud M, and Fevre M. 1990. Purification and characterization of an extracellular betaxylosidase from the rumen anaerobic fungus Neocallimastix frontalis. FEMS Microbiol

601 Lett 72:11-16. DOI 10.1111/j.1574-6968.1990.tb03853.x

602 Hess M, Sczyrba A, Egan R, Kim TW, Chokhawala H, Schroth G, Luo SJ, Clark DS, Chen F, 603 Zhang T, Mackie RI, Pennacchio LA, Tringe SG, Visel A, Woyke T, Wang Z, and Rubin 604 EM. 2011. Metagenomic discovery of biomass-degrading genes and genomes from cow 605 rumen. Science 331:463-467. 10.1126/science.1200387 
606 Hill J, Nelson E, Tilman D, Polasky S, and Tiffany D. 2006. Environmental, economic, and 607 energetic costs and benefits of biodiesel and ethanol biofuels. P Natl Acad Sci USA

608 103:11206-11210. 10.1073/pnas.0604600103

609 Hinz SWA, van den Broek LAM, Beldman G, Vincken JP, and Voragen AGJ. 2004. Beta-

610 galactosidase from Bifidobacterium adolescentis DSM20083 prefers beta(1,4)-

611 galactosides over lactose. Appl Microbiol Biot 66:276-284. 10.1007/s00253-004-1745-9

612 Hovel K, Shallom D, Niefind K, Belakhov V, Shoham G, Baasov T, Shoham Y, and Schomburg D. 2003. Crystal structure and snapshots along the reaction pathway of a family 51 alphaL-arabinofuranosidase. EMBO J 22:4922-4932. Doi 10.1093/Emboj/Cdg494

John M, Schmidt B, and Schmidt J. 1979. Purification and some properties of five endo-1,4-betaD-xylanases and a beta-D-xylosidase produced by a strain of Aspergillus niger. Can J Biochem Cell B 57:125-134.

618 Kubicek CP. 1982. Beta-glucosidase excretion by Trichoderma pseudokoningii - correlation with 619 cell-wall bound beta-1.3-glucanase activities. Arch Microbiol 132:349-354. Doi

620 10.1007/Bf00413388

621

622

623

624

625

626

627 628
Kumar R, Singh S, and Singh OV. 2008. Bioconversion of lignocellulosic biomass: biochemical and molecular perspectives. J Ind Microbiol Biot 35:377-391. 10.1007/s10295-008-03278

Kumar S, and Ramon D. 1996. Purification and regulation of the synthesis of a beta-xylosidase from Aspergillus nidulans. FEMS Microbiol Lett 135:287-293. DOI 10.1111/j.15746968.1996.tb08003.x

Laemmli UK. 1970. Cleavage of Structural Proteins during Assembly of Head of BacteriophageT4. Nature 227:680-\&. Doi 10.1038/227680a0 
629 Liggenstoffer AS, Youssef NH, Wilkins MR, and Elshahed MS. 2014. Evaluating the utility of

630 hydrothermolysis pretreatment approaches in enhancing lignocellulosic biomass

631 degradation by the anaerobic fungus Orpinomyces sp. strain C1A. J Microbiol Meth

$632 \quad 104: 43-48.10 .1016 /$ j.mimet.2014.06.010

633 Lineweaver H, and Burk D. 1934. The determination of enzyme dissociation constants. $J$ Am

$634 \quad$ Chem Soc 56:658-666. Doi 10.1021/Ja01318a036

635 Liu ZL, Saha BC, and Slininger PJ. 2008. Lignocellulosic biomass conversion to ethanol by

636 Saccharomyces. In: Harwood CS, Demain AL, and Wall JD, eds. Bioenergy. Washington

637 D.C.: ASM Press.

638 Ljungdahl LG. 2008. The cellulase/hemicellulase system of the anaerobic fungus Orpinomyces

639 PC-2 and aspects of its applied use. Ann Ny Acad Sci 1125:308-321.

$640 \quad 10.1196 /$ annals. 1419.030

641 Lombard V, Ramulu HG, Drula E, Coutinho PM, and Henrissat B. 2014. The carbohydrate-

642 active enzymes database (CAZy) in 2013. Nucleic Acids Res 42:D490-D495.

$643 \quad 10.1093 /$ nar/gkt1 178

644 Matsuo M, and Yasui T. 1984. Purification and some properties of beta-xylosidase from

645 Trichoderma viride. Agr Biol Chem 48:1845-1852.

646 Morrison JM, Elshahed MS, and Youssef NH. 2016. A defined enzyme cocktail from the

647 anaerobic fungus Orpinomyces sp. strain C1A effectively releases sugars from pretreated

$648 \quad$ corn stover and switchgrass. Sci Rep Accepted.

649 Morrison JM, Wright CM, and John GH. 2012. Identification, isolation and characterization of a 650 novel azoreductase from Clostridium perfringens. Anaerobe 18:229-234.

$651 \quad$ 10.1016/j.anaerobe.2011.12.006 
652 National Research Council N. 2011. Renewable Fuel Standard: Potential Economic and

653 Environmental Effects of U.S. Biofuel Policy. Washington, D.C.

654 PyMol. The PyMOL Molecular Graphics System. Version 1.7.4 ed: Schrodinger, LLC.

655 Ragauskas AJ, Williams CK, Davison BH, Britovsek G, Cairney J, Eckert CA, Frederick WJ, 656 Hallett JP, Leak DJ, Liotta CL, Mielenz JR, Murphy R, Templer R, and Tschaplinski T.

657

658 2006. The path forward for biofuels and biomaterials. Science 311:484-489.

659

660 10.1126/science. 1114736

Roy A, Kucukural A, and Zhang Y. 2010. I-TASSER: a unified platform for automated protein structure and function prediction. Nat Protoc 5:725-738. 10.1038/nprot.2010.5

661

662

663

664

665

666

667

668

669

670

671

672

673 Skalova T, Dohnalek J, Spiwok V, Lipovova P, Vondrackova E, Petrokova H, Duskova J, Strnad

674

10.1146/annurev-arplant-042809-112315

Shao WL, Xue YM, Wu AL, Kataeva I, Pei JJ, Wu HW, and Wiegel J. 2011. Characterization of a bovel beta-xylosidase, XylC, from Thermoanaerobacterium saccharolyticum JW/SLYS485. Appl Environ Microbiol 77:719-726. 10.1128/Aem.01511-10

Sievers F, Wilm A, Dineen D, Gibson TJ, Karplus K, Li WZ, Lopez R, McWilliam H, Remmert M, Soding J, Thompson JD, and Higgins DG. 2011. Fast, scalable generation of highquality protein multiple sequence alignments using Clustal Omega. Mol Syst Biol 7:1-6. Artn 539 10.1038/Msb.2011.75

H, Kralova B, and Hasek J. 2005. Cold-active beta-galactosidase from Arthrobacter sp 
C2-2 forms compact $660 \mathrm{kDa}$ hexamers: crystal structure at 1.9 angstrom resolution. $J$

676 Mol Biol 353:282-294. 10.1016/j.jmb.2005.08.028

677 Stamatakis A. 2014. RAxML version 8: a tool for phylogenetic analysis and post-analysis of 678 large phylogenies. Bioinformatics 30:1312-1313. 10.1093/bioinformatics/btu033

679 Takashima S, Iikura H, Nakamura A, Hidaka M, Masaki H, and Uozumi T. 1998.

680 Overproduction of recombinant Trichoderma reesei cellulases by Aspergillus oryzae and 681 their enzymatic properties. J Biotechnol 65:163-171. Doi 10.1016/S0168-1656(98)000846824

683 Tamura K, Stecher G, Peterson D, Filipski A, and Kumar S. 2013. MEGA6: molecular 684 evolutionary genetics analysis version 6.0. Mol Biol Evol 30:2725-2729.

$685 \quad 10.1093 / \mathrm{molbev} / \mathrm{mst} 197$

686 Tong CC, Cole AL, and Shepherd MG. 1980. Purification and properties of the cellulases from 687 the thermophilic fungus Thermoascus aurantiacus. Biochem J 191:83-94.

688 Ulrich JT, Temple KL, and Mcfeters GA. 1972. Induction and characterization of beta689 galactosidase in an extreme thermophile. J Bacteriol 110:691-698.

690 Van Dyk JS, and Pletschke BI. 2012. A review of lignocellulose bioconversion using enzymatic 691 hydrolysis and synergistic cooperation between enzymes-Factors affecting enzymes, 692 conversion and synergy. Biotechnol Adv 30:1458-1480.

$693 \quad$ 10.1016/j.biotechadv.2012.03.002

694 vanPeij NNME, Brinkmann J, Vrsanska M, Visser J, and deGraaff LH. 1997. Beta-xylosidase 695 activity, encoded by $x \operatorname{lnD}$, is essential for complete hydrolysis of xylan by Aspergillus 696 niger but not for induction of the xylanolytic enzyme spectrum. Eur J Biochem 245:164$697 \quad 173$. 
698 Verdoucq L, Moriniere J, Bevan DR, Esen A, Vasella A, Henrissat B, and Czjzek M. 2004.

699 Structural determinants of substrate specificity in family 1 beta-glucosidases - Novel

700 insights from the crystal structure of sorghum dhurrinase-1, a plant beta-glucosidase with

$701 \quad$ strict specificity, in complex with its natural substrate. J Biol Chem 279:31796-31803.

702 10.1074/jbc.M402918200

703 Wagschal K, Franqui-Espiet D, Lee CC, Robertson GH, and Wong DWS. 2008. Cloning, 704

705

706 expression and characterization of a glycoside hydrolase family 39 xylosidase from Bacillus halodurans C-125. Appl Biochem Biotech 146:69-78. 10.1007/s12010-007-

707 Wang HC, Chen YC, Huang CT, and Hseu RS. 2013. Cloning and characterization of a 708 thermostable and pH-stable cellobiohydrolase from Neocallimastix patriciarum J11.

709 Protein Expres Purif 90:153-159. 10.1016/j.pep.2013.06.004

710 Yang JK, Yoon HJ, Ahn HJ, Lee BI, Pedelacq JD, Liong EC, Berendzen J, Laivenieks M, Vieille 711 C, Zeikus GJ, Vocadlo DJ, Withers SG, and Suh SW. 2004. Crystal structure of beta-D-

712 xylosidase from Thermoanaerobacterium saccharolyticum, a family 39 glycoside hydrolase. J Mol Biol 335:155-165. 10.1016/j.jmb.2003.10.026

714 Yang JY, Yan RX, Roy A, Xu D, Poisson J, and Zhang Y. 2015. The I-TASSER Suite: protein 715 structure and function prediction. Nat Methods 12:7-8. 10.1038/nmeth.3213

716 Youssef NH, Couger MB, Struchtemeyer CG, Liggenstoffer AS, Prade RA, Najar FZ, Atiyeh

717 HK, Wilkins MR, and Elshahed MS. 2013. The genome of the anaerobic fungus

718 Orpinomyces sp strain C1A reveals the unique evolutionary history of a remarkable plant 719 biomass degrader. Appl Environ Microbiol 79:4620-4634. 10.1128/Aem.00821-13 
720 Yuan TZ, Yang PL, Wang YR, Meng K, Luo HY, Zhang W, Wu NF, Fan YL, and Yao B. 2008.

721 Heterologous expression of a gene encoding a thermostable beta-galactosidase from

$722 \quad$ Alicyclobacillus acidocaldarius. Biotechnol Lett 30:343-348. 10.1007/s10529-007-9551-

$723 \quad \mathrm{y}$

724 Zhang Y. 2008. I-TASSER server for protein 3D structure prediction. BMC Bioinformatics 9:40-

725 48. Artn 40 10.1186/1471-2105-9-40

726 Zhang YHP, Hong J, and Xinhao Y. 2009. Cellulase Assays. In: Mielenz JR, ed. Biofuels:

$727 \quad$ Methods and Protocols: Humana Press, 213-231.

728 Zhu H, Cheng KJ, and Forsberg CW. 1994. A truncated beta-xylosidase from the anaerobifungus

729 Neocallimastix patriciarum 27. Can J Microbiol 40:484-490.

730

731

732

733

734

735

736

737

738

739

740

741

742 


\section{$743 \quad$ Figure Legends}

744 Figure 1. Phylogenetic analysis of GH39 $\beta$-xylosidases, including Bgxg1. Sequences annotated as GH39 $\beta$ -

745 xylosidases ( $\mathrm{n}=200$ sequences, October 28, 2015) were retrieved from CAZyme databases (Lombard et al. 2014).

746 Genbank accession numbers are shown for reference proteins (due to the unavailability of Piromyces proteins in

747 Genbank, those proteins are shown as JGI accession numbers). The Maximum Likelihood tree was generated in

748 RAxML (Stamatakis 2014) using a BLOSUM62 substitution matrix and a GAMMA model of rate heterogeneity.

749 The model estimated an alpha parameter of 2.069. Bootstraps values (100 replicates) are shown for nodes with $>50$

750 bootstrap support. The sequences were empirically classified into four classes (Classes I-IV), and Class III, to which

751 Bgxg1 is affiliated, is further classified into four distinct lineages (III-A-III-D). The $\alpha$-iduronidase sequence from

752 Mus musculus was utilized as an outgroup. $\beta$-xylosidases that were previously characterized biochemically were

753 phylogenetically affiliated with either Class II (Bacillus halodurans (BAB04787.1) and Geobacillus

754 stearothermophilus (ABI49941.1) in bottom Firmicutes wedge, and Thermoanaerobacterium saccharolyticum

755 (AAB68820.1) in middle Firmicutes wedge) or Class I (Caulobacter crescentus (ACL95907.1), bottom $\alpha$ -

756 Proteobacteria wedge). Bgxg1, from Orpinomyces sp. strain C1A, is shown highlighted in yellow.

757

758 Figure 2. Effect of Temperature and pH on Bgxg1 activity A) Optimal pH, B) Optimal Temperature, C) pH

759 Stability, D) Thermal Stability. All values are presented as relative specific activities, calculated by determining

760 the \% activity relative to the highest activity (with the highest activity set at 100\%). For A, B, C, and D, PNPX was

761 used as a substrate. Error bars represent standard deviation of triplicate $(n=3)$ samples.

762

763 Figure 3. Substrate competition and Bgxg1 preference. Monosaccharides (glucose (ם) or xylose (\&s)) release

764 was assayed when Bgxg1 was challenged with $10 \mathrm{mM}$ cellobiose (A), $10 \mathrm{mM}$ xylobiose (B), or an equimolar

765 mixture of both substrates $(\mathbf{C})$. In (A), the effect of xylobiose (as a competitive inhibitor) is measured through

766 conducting a $\beta$-glucosidase activity assay. In (B), the effect of cellobiose (as a competitive inhibitor) is measured

767 through conducting a $\beta$-xylosidase activity assay. In $(\mathbf{C})$, a competition assay was performed with both cellobiose

768 and xylobiose present, assaying for the presence of glucose or xylose.

769 
770 Figure 4. Alignment of Bgxg1 and the four biochemically-characterized GH39-family enzymes, highlighting

771 structural predictions and conservation of or around the active site. Structural predictions for Bgxg1 sequence

772 were obtained using I-TASSER three-dimensional model (Fig. S3) (Roy et al. 2010; Yang et al. 2015; Zhang 2008).

773 Bgxg1 sequence is compared to those from Caulobacter crescentus, Thermoanaerobacterium saccharolyticum,

774 Geobacillus stearothermophilus, and Bacillus halodurans. $\alpha$-helices in blue are those within the small $\alpha$-helical

775 domain, $\alpha$-helices and $\beta$-sheets in green are those within the $(\alpha / \beta)_{8}$ barrel, and $\beta$-sheets in red are those within the $\beta$ -

776 sandwich. Red stars $(*)$ represent catalytic residues within the active site. Black stars $(*)$ represent those residues

777 close to the active site, as determined within the Bgxg1 model. Blue stars $(*)$ represent residues noted in the

778 literature to be important for $\beta$-xylosidase function (Czjzek et al. 2005; Yang et al. 2004).

779 


\section{Table 1 (on next page)}

Table 1

Table 1. Substrate Specificity and Specific Activity of Bgxg1 
1 Table 1. Substrate Specificity and Specific Activity of Bgxg1.

\begin{tabular}{lll}
\hline Substrate $^{\mathbf{a}}$ & Activity Tested & $\begin{array}{l}\text { Specific Activity } \\
(\mathbf{U} / \mathbf{m g} \pm \text { SD) }\end{array}$ \\
\hline PNPG & $\beta$-glucosidase & $73.4 \pm 7.15$ \\
Cellobiose & $\beta$-glucosidase & $55.1 \pm 5.36$ \\
PNPGal & $\beta$-galactosidase & $54.6 \pm 2.26$ \\
PNPX & $\beta$-xylosidase & $11.5 \pm 1.2$ \\
Xylobiose & $\beta$-xylosidase & $10.9 \pm 0.96$ \\
Beechwood Xylan & Xylanase & $10.8 \pm 1.25$ \\
Avicel & Exoglucanase & $\mathrm{ND}^{\mathbf{b}}$ \\
CMC & Endoglucanase & $\mathrm{ND}^{\mathbf{b}}$ \\
Locust Bean Gum & Mannanase & $\mathrm{ND}^{\mathbf{b}}$ \\
PNPA & Arabinosidase & $\mathrm{ND}^{\mathbf{b}}$ \\
PNPAc & Acetyl Xylan Esterase & $\mathrm{ND}^{\mathbf{b}}$ \\
PNPC & Cellobiohydrolase & $\mathrm{ND}^{\mathbf{b}}$ \\
PNPM & Mannosidase & $\mathrm{ND}^{\mathbf{b}}$ \\
Aldouronic acid & $\alpha$-glucuronidase & $\mathrm{ND}^{\mathbf{b}}$ \\
\hline
\end{tabular}

2 a Abbreviations: PNPC - $p$-nitrophenyl- $\beta$-D-cellobioside, PNPX - $p$-nitrophenyl- $\beta$-D-xylopyranoside, PNPA - $p$ -

3 nitrophenyl- $\beta$-D-arabinofuranoside, PNPM - $p$-nitrophenyl- $\beta$-D-mannoside, PNPG - $p$-nitrophenyl- $\beta$-D-

4 glucopyranoside, PNPGal - $p$-nitrophenyl- $\beta$-D-galactopyranoside, PNPAc - $p$-nitrophenyl-acetate.

5 b ND: Not detected. 
Table 2 (on next page)

Enzyme Kinetics for Bgxg1 
1 Table 2. Enzyme Kinetics for Bgxg1. $K_{m}$ and $V_{\max }$ values were calculated by extrapolation from Lineweaver-Burke 2 plots.

\begin{tabular}{lllc}
\hline Substrate $^{\mathbf{a}}$ & Activity Tested & $\boldsymbol{K}_{\boldsymbol{m}}^{\mathbf{b}}$ & $\boldsymbol{V}_{\boldsymbol{m a x}}(\mathbf{U} / \mathbf{m g})$ \\
\hline PNPG & $\beta$-glucosidase & $\mathrm{BDL}^{\mathrm{c}}$ & $769 \pm 18$ \\
PNPGal & $\beta$-galactosidase & $\mathrm{BDL}^{\mathrm{d}}$ & $769 \pm 13$ \\
PNPX & $\beta$-xylosidase & $0.00485 \mathrm{mM} \pm 0.00062$ & $127 \pm 8$ \\
Beechwood Xylan & Xylanase & $0.038 \mathrm{mg} / \mathrm{mL} \pm 0.0039$ & $25.6 \pm 10$ \\
\hline
\end{tabular}

3 a: Abbreviations: PNPG - $p$-nitrophenyl- $\beta$-D-glucopyranoside, PNPGal - $p$-nitrophenyl- $\beta$-D-galactopyranoside,

4 PNPX - $p$-nitrophenyl- $\beta$-D-xylopyranoside,.

5 b: $K_{m}$ values are expressed in either $\mathrm{mM}$ or $\mathrm{mg} / \mathrm{mL}$, depending on the substrate tested. Values are shown \pm standard

6 deviation of triplicate samples $(\mathrm{n}=3)$.

7 c: BDL: Below detection limit $(500 \mathrm{nM})$. Extrapolated $K_{m}$ value obtained using Lineweaver-Burke plot was

$80.0000125 \mathrm{mM} \pm 0.0000096$.

9 d: BDL: Below detection limit $(500 \mathrm{nM})$. Extrapolated $K_{m}$ value obtained using Lineweaver-Burke plot was

$100.000214 \mathrm{mM} \pm 0.000016$.

11 
Table 3 (on next page)

Substrate competition experiments. 
2 Table 3. Substrate competition experiments. "Activity tested" column refers to the colorimetric substrate tested

3 (PNPX for $\beta$-xylosidase, PNPG for $\beta$-glucosidase) in the presence of the active site inhibitor (cellobiose or

4 xylobiose, at listed Inhibitor concentrations). Specific activity, $K_{m}$, and $V_{\max }$ refer to the values calculated for the

5 colorimetric substrate in each experiment.

\begin{tabular}{cccccc}
\hline $\begin{array}{c}\text { Activity } \\
\text { Tested }\end{array}$ & $\begin{array}{c}\text { Active Site } \\
\text { Inhibitor }\end{array}$ & $\begin{array}{c}\text { Inhibitor } \\
(\mathbf{m M})\end{array}$ & $\begin{array}{c}\text { Relative Specific } \\
\text { Activity }(\mathbf{\%})\end{array}$ & $\begin{array}{c}\boldsymbol{K}_{\boldsymbol{m}} \\
(\mathbf{m M})\end{array}$ & $\begin{array}{c}\boldsymbol{V}_{\max } \\
(\mathbf{U} / \mathbf{m g})\end{array}$ \\
\hline$\beta$-xylosidase & Cellobiose & 0 & 100 & 0.00485 & 127 \\
& & 10 & 78.9 & 1.438 & 118 \\
$\beta$-glucosidase & Xylobiose & 20 & 52.1 & 3.51 & 129 \\
& & 10 & 100 & 0.0000125 & 769 \\
& & 20 & 75.8 & 0.000235 & 763 \\
& & 57.2 & 0.00349 & 752 \\
\hline
\end{tabular}

6

7 


\section{Table 4 (on next page)}

Comparison of key amino acids between Bgxg1 and all four biochemically characterized $\beta$-xylosidases 
1 Table 4. Comparison of key amino acids between Bgxg1 and all four biochemically characterized (BC) $\beta$ -

2 xylosidases from Thermoanaerobacterium saccharolyticum, Bacillus halodurans, Geobacillus

3 stearothermophilus, Caulobacter crescentus, as well as in Classes III-A, III-B, and III-Da.

\begin{tabular}{|c|c|c|c|c|c|c|c|c|}
\hline Pos. ${ }^{b}$ & $\begin{array}{l}\text { AA in } \\
\text { Bgxg1 }\end{array}$ & $\begin{array}{c}\mathrm{AA} \text { in } 4 \\
\mathrm{BC}^{\mathrm{d}}\end{array}$ & Significance of Change & $\begin{array}{l}\text { Importance } \\
\text { of Residue }\end{array}$ & $\begin{array}{l}\text { Class } \\
\text { III-A }\end{array}$ & $\begin{array}{l}\text { Class } \\
\text { III-B }\end{array}$ & $\begin{array}{l}\text { Class } \\
\text { III-C }\end{array}$ & $\begin{array}{l}\text { Class } \\
\text { III-D }\end{array}$ \\
\hline 46 & Val & Tyr & $\begin{array}{l}\text { Small, nonpolar (Val) vs. Large, } \\
\text { polar (Tyr) }\end{array}$ & $\begin{array}{l}\text { Near active } \\
\text { site }\end{array}$ & $\mathrm{NC}^{\mathbf{e}}$ & Ile & Val & $\mathrm{NC}$ \\
\hline 129 & Asp & Asn & $\begin{array}{l}\text { Negative charge (Asp) vs. Neutral } \\
\text { charge (Asn) }\end{array}$ & H-bonding & Lys & Asp & Asp & Asp \\
\hline $131^{\mathbf{c}}$ & $\mathrm{Thr} / \mathrm{NC}$ & Phe & $\begin{array}{l}\text { Small, polar (Thr) vs. Large, } \\
\text { nonpolar (Phe) }\end{array}$ & $\begin{array}{l}\text { Near active } \\
\text { site }\end{array}$ & $\mathrm{NC}$ & $\mathrm{NC}$ & $\mathrm{NC}$ & $\mathrm{NC}$ \\
\hline 139 & Phe & Tyr & $\begin{array}{l}\text { Large, nonpolar (Phe) vs. Large, } \\
\text { polar (Tyr) }\end{array}$ & $\begin{array}{l}\text { Near active } \\
\text { site }\end{array}$ & Tyr & Tyr & Phe & Tyr \\
\hline 163 & Cys & Ala & $\begin{array}{l}\text { Polar, thiol (Cys) vs. Nonpolar } \\
\text { (Ala) }\end{array}$ & $\begin{array}{l}\text { Near active } \\
\text { site }\end{array}$ & Tyr & Ala & Cys & Tyr \\
\hline 171 & Lys & Trp & $\begin{array}{l}\text { Positive charge (Lys) vs. } \\
\text { Nonpolar (Trp) }\end{array}$ & $\begin{array}{l}\text { Near active } \\
\text { site }\end{array}$ & $\operatorname{Trp}$ & $\mathrm{NC}$ & Lys & Lys \\
\hline 194 & Leu & Tyr & $\begin{array}{l}\text { Small, nonpolar (Leu) vs. Large, } \\
\text { polar (Tyr) }\end{array}$ & H-bonding & Ser & Ile/Glu & Leu & Tyr \\
\hline 242 & Arg & Ala & $\begin{array}{l}\text { Positive charge (Arg) vs. Small, } \\
\text { nonpolar (Ala) }\end{array}$ & $\begin{array}{l}\text { Near active } \\
\text { site }\end{array}$ & $\mathrm{NC}$ & $\mathrm{NC}$ & Arg & $\mathrm{NC}$ \\
\hline $322-323$ & -gap- & Glu & Gap vs. Negative charge (Glu) & H-bonding & Arg/Thr/Lys & -gap- & -gap- & -gap- \\
\hline $322-323$ & -gap- & Glu & Gap vs. Negative charge (Glu) & H-bonding & Gly/-gap- & -gap- & -gap- & -gap- \\
\hline
\end{tabular}

4

5 a No changes were identified in 10 different positions (Arg48, Ile124, Trp125, Asn126, Glu127, Pro128, Trp132,

6 Pro162, His192, Glu225), and 5 positions were variable across all sequences (Val81, Gly130, Tyr164, Ser165,

7 Lys247).

8 b Pos. (Positions) refer to the position of the amino acid in Bgxg1

$9 \mathbf{c}^{\mathbf{c}}$ Bgxg1 and all proteins in Class III-C $\beta$-xylosidases have identical amino acid sequences in all key positions with 10 one exception (Thr131)

$11 \mathrm{~d}$ Sequences identified using the alignment presented in Fig. 5.

$12{ }^{\mathbf{e}} \mathrm{NC}=$ Not conserved 
Figure 1 (on next page)

Phylogenetic analysis of GH39 $\beta$-xylosidases, including Bgxg1. 


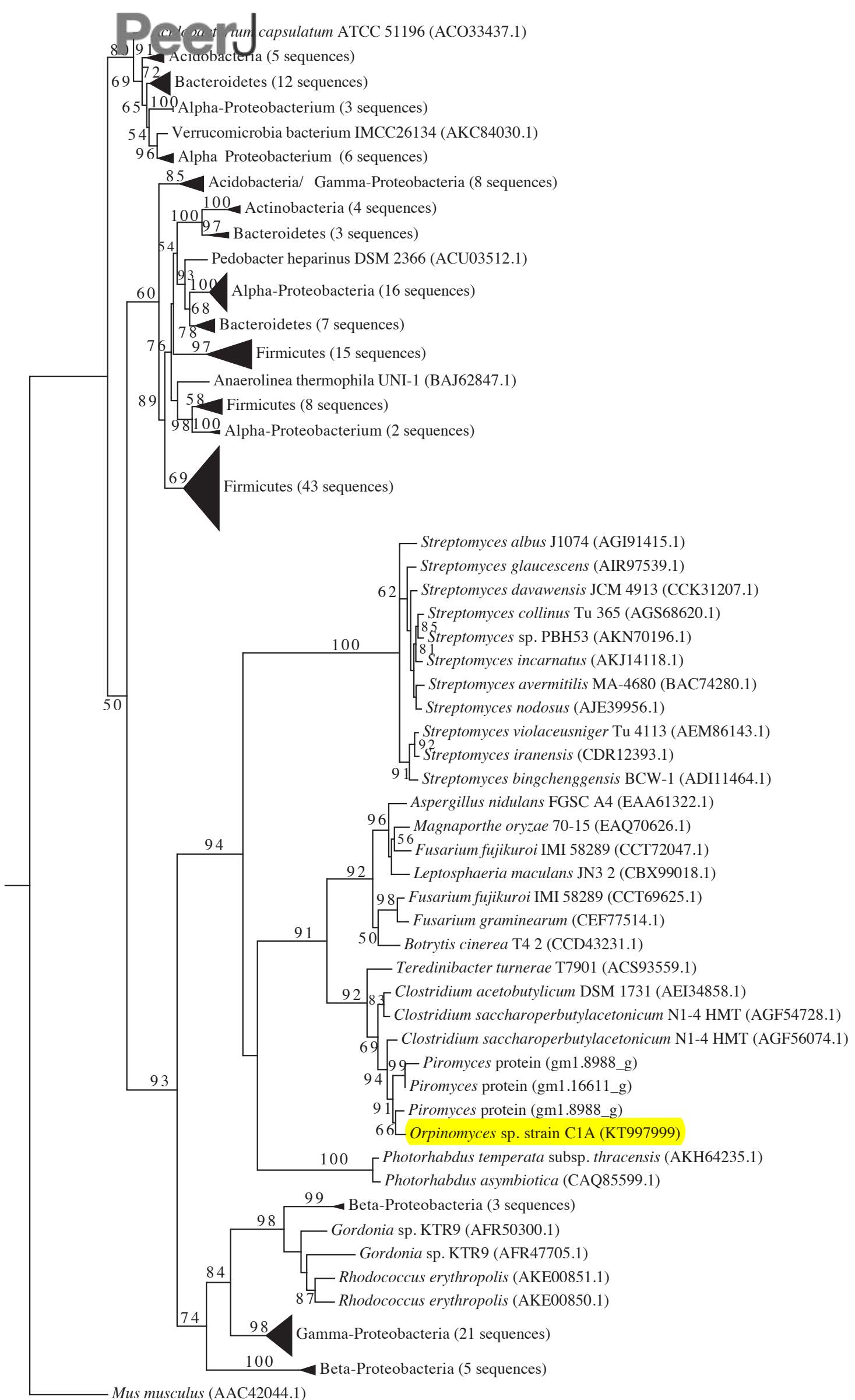


Figure 2 (on next page)

Effect of Temperature and pH on Bgxg1 activity A) Optimal pH, B) Optimal Temperature, C) pH Stability, D) Thermal Stability 

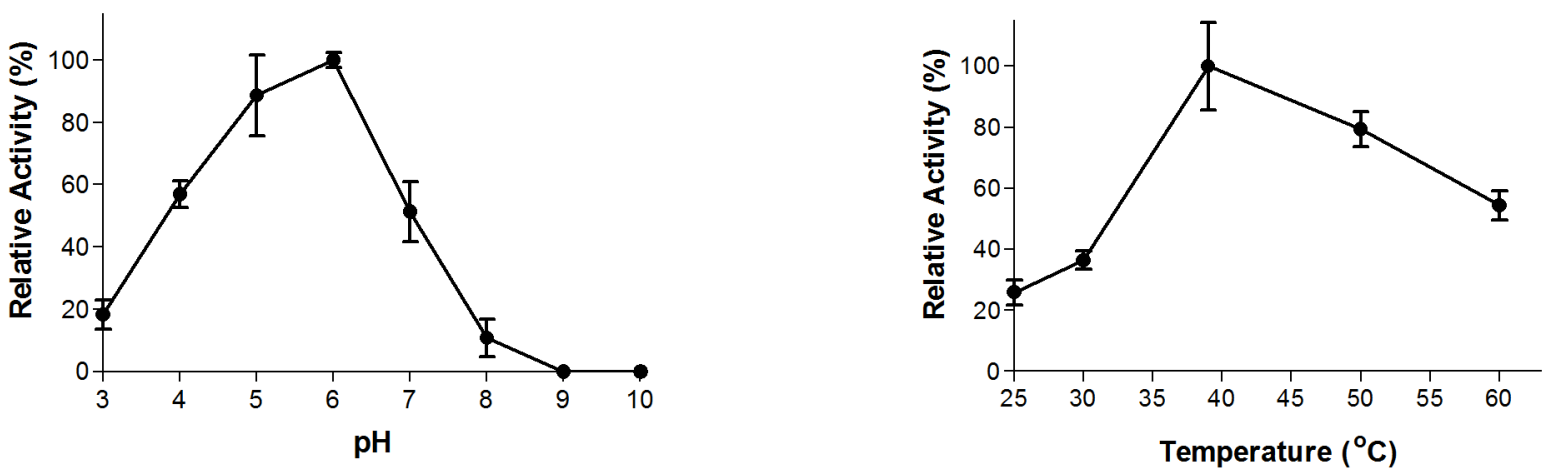

C

D
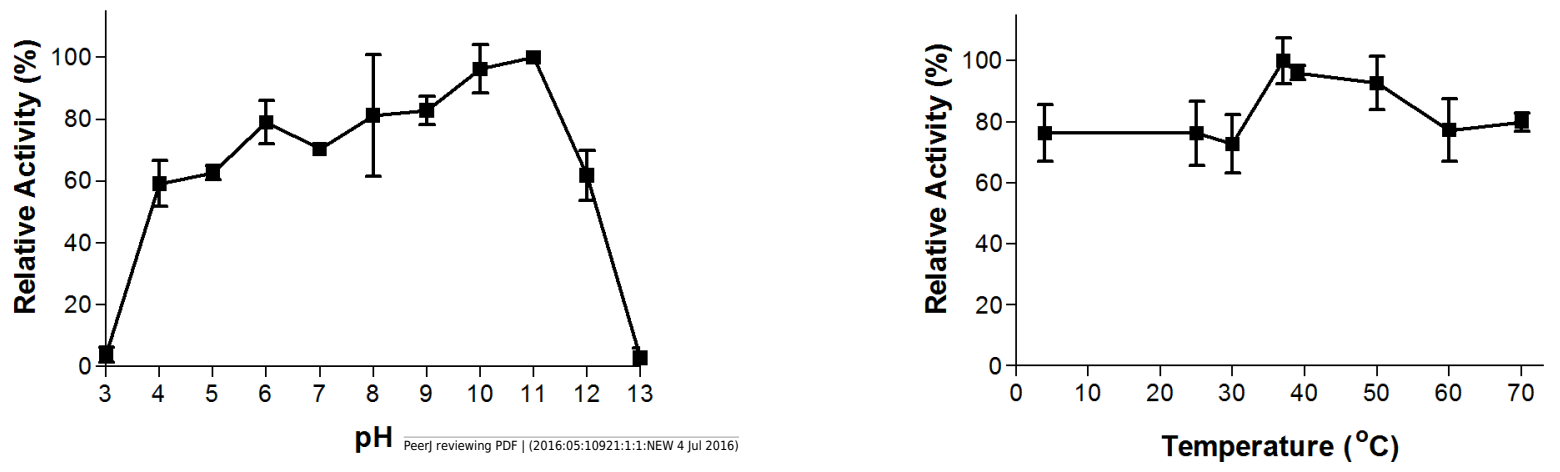
Figure 3

Alignment of Bgxg1 and the four biochemically-characterized GH39-family enzymes, highlighting structural predictions and conservation of or around the active site. 

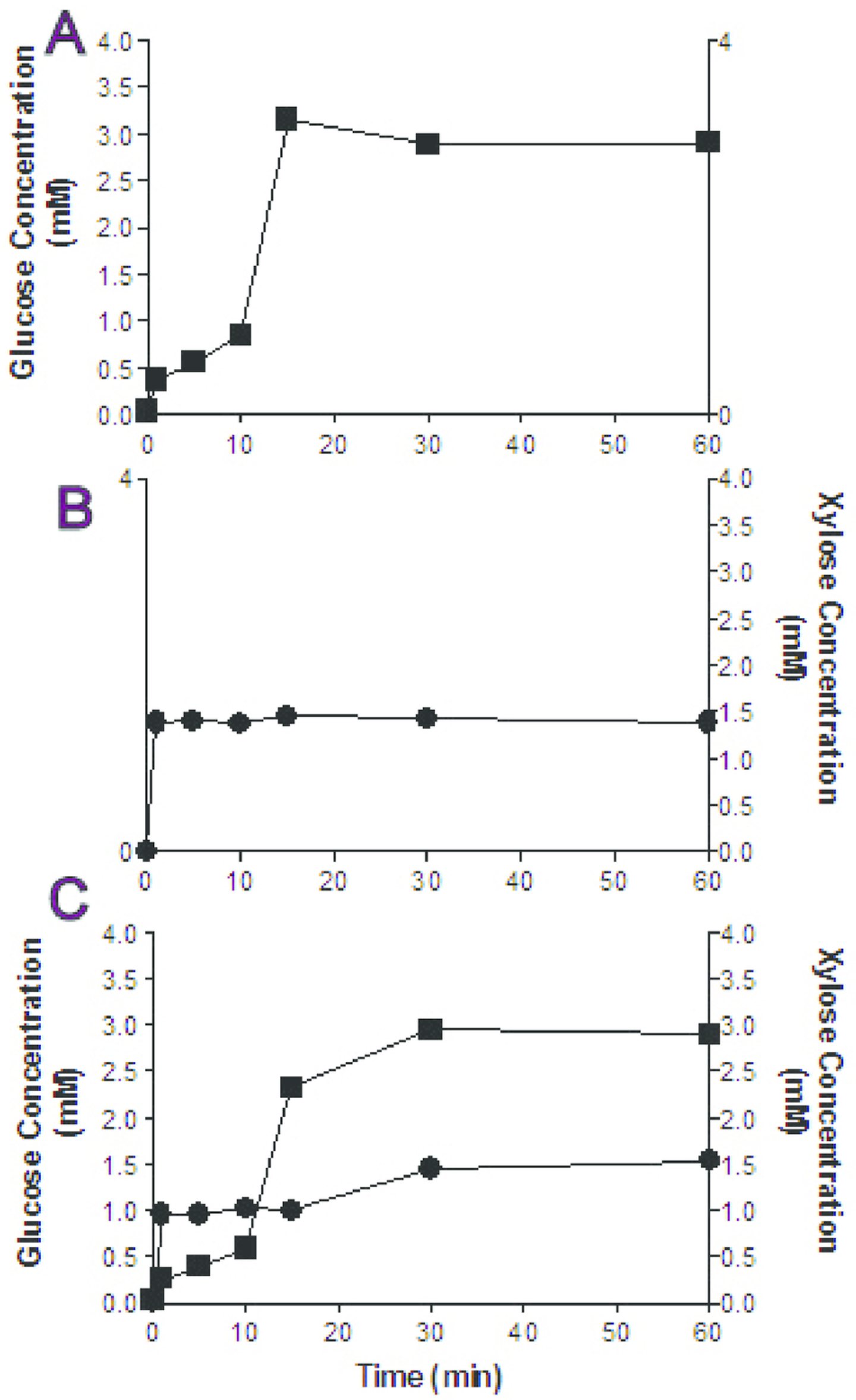
Figure 4 (on next page)

Alignment of Bgxg1 and the four biochemically-characterized GH39-family enzymes, highlighting structural predictions and conservation of or around the active site. 
Bgxg1

Caulobacter

Thermoanaerobacterium Geobacillus

Bacillus

Bgxg1

Caulobacter

Thermoanaerobacterium Geobacillus

Bacillus

Bgxg1

Caulobacter

Thermoanaerobacterium Geobacillus

Bacillus

$\operatorname{Bg} x g 1$

Caulobacter

Thermoanaerobacterium Geobacillus

Bacillus

Bgxg1

Caulobacter

Thermoanaerobacterium Geobacillus

Bacillus

Bgxg1

Caulobacter

Thermoanaerobacterium Geobacillus

Bacillus

Bgxg1

Caulobacter

Thermoanaerobacterium Geobacillus Bacillus

1 MT . . . . . N V LTV E . . CNNKL . RRATHCANGSLYGIT . ETTPRDYKNLVDPLHPFVMRN . PARGGNG

1 MANAGPGAR VIDLDLRRAAGPVDRFFDLSIGSDYPGTLIREDSOAOLKTTVDELGFR YIRFHAIFHDVLGTVKVQ . . . . .

1

1

1

$M_{I K} V_{R} V_{P}$. DFSDKKFSDRWRYCVGTGRLGLALQKEYIETLKYVKENIDFKYIRGHGLLCDDVGIYRED . VVGD $M_{K} V_{N} V_{P}$. SNGREKF KKNWKFCVGTGRLGLALQKEYLDHLKLVQEKIGFRYIRGHGLLSDDVGIYREV . EIDG

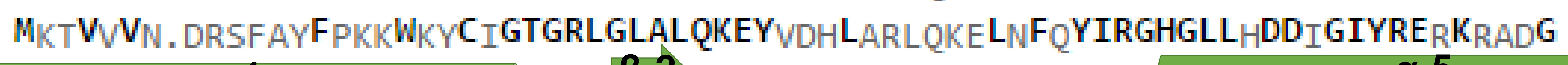
$\alpha-4$

3-2 $\alpha-5$

57 NQHPYGDAI . . . . . . . . . . KVARRLADTPGALVSVDLPDMLPGWPYKWPG . . . . . MQNWLNQVKSFI . . . KDK

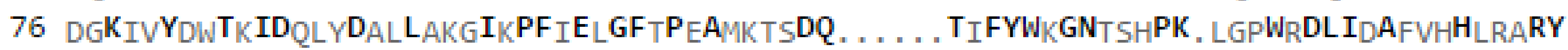

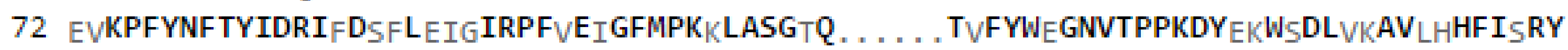

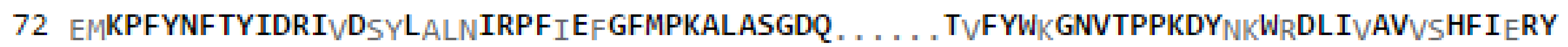

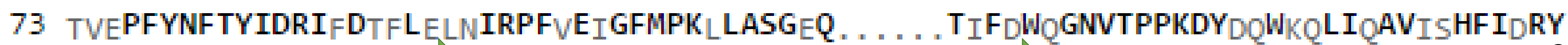
$\alpha-5 \quad \beta-3, \alpha-6 \quad \beta-4$

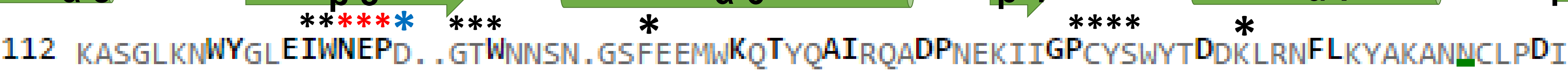

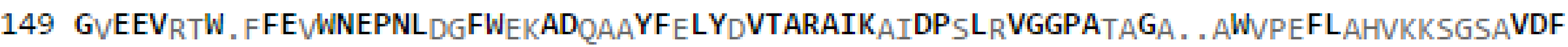

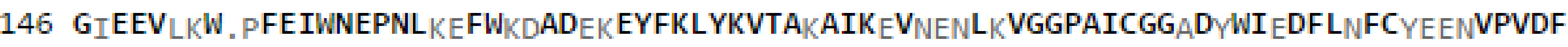

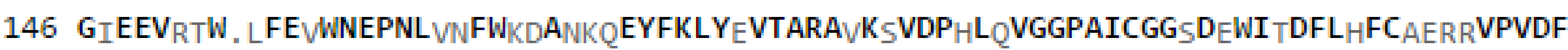

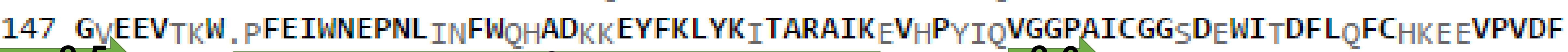

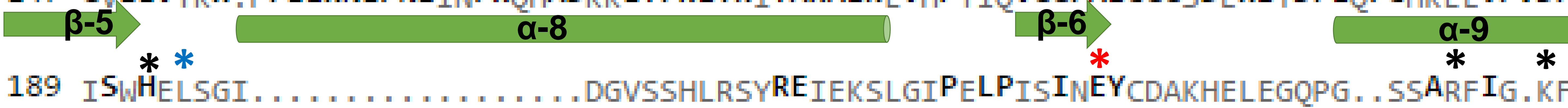

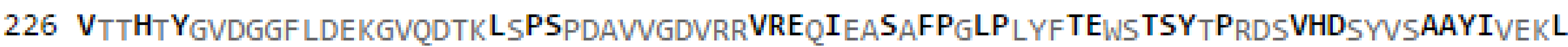

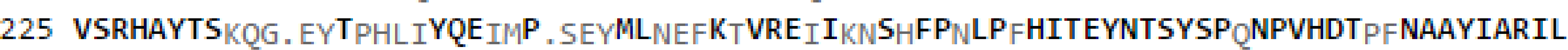

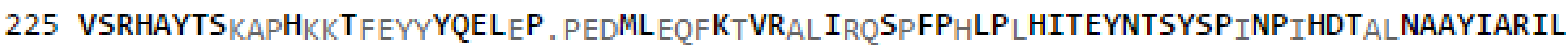

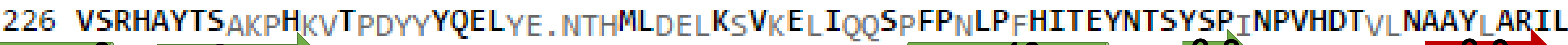
$\alpha-9 \quad \beta-7>* *$ $\alpha-10 \quad \beta-8$

248 ERYKV. DTAMITWWFV . . . . . . . . . PLPGRLGSL LATDTQKGAGWYFYKWYGDMTGDMVYVKPPNDNSNLVDGAACVD 306 RRVKGLV V VMSYWTYSDLFEEPGPPTAPF GGFG , LMNPQG IRKPSWFAYKYLNALKGRELVCADDOVFAARDGDRVA . . 303 SEGGVVDSFSYWTFSDVFEERDVPRSQFHGGFG, LVALMMIPKPT FYTFKFFNAMGEEMLYRDEHMLVTRRDDGSVA .

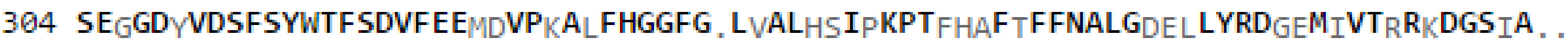

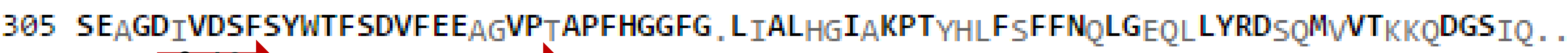
$\beta-10$

316 TNKEYISFIFGGPNDGTI . RASSIIFQALLDL $L$ PML

$383 \ldots$... IVAYAWRQPDQKVSNRPFYTKLHPASDV EPLKVRLTSLKPGRYKLRVRRVGYRRNDAYSAYIDMGSPTTLTESQLQ $380 \ldots$... . . . AWNEVMDKTENPDEDYEVE .... . IPVRF . . . . . . RDVFIKRQLIDEEHGNPWGTWIHMGRPRYPSKEQVN

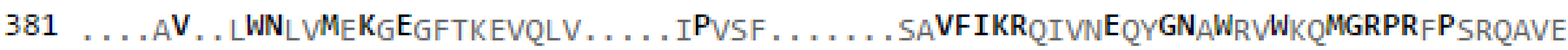

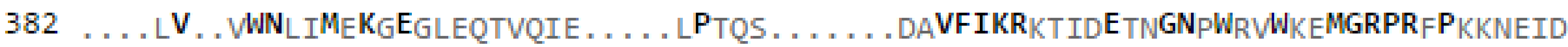

351

459 SLQALTEDRPEIEKALVSGETVVDLPMRANDVVLIELEPLA

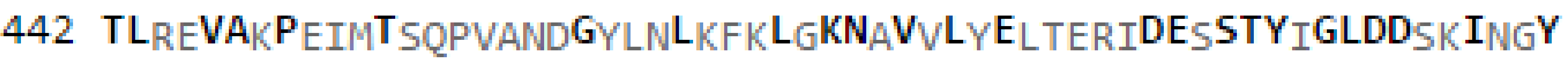

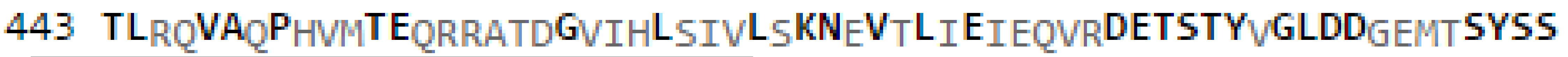

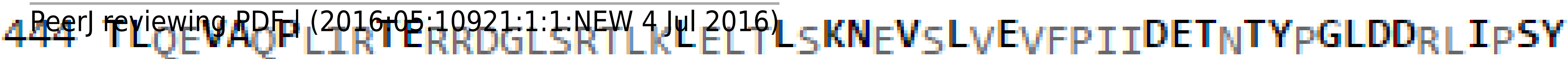

